\title{
Aggregation of individual rankings through fusion functions: criticism and optimality analysis
}

\author{
H. Bustince, Senior Member, IEEE B. Bedregal, M. J. Campión, I. Da Silva, J. \\ Fernandez, Member, IEEE E. Induráin, A. Raventós-Pujol, R.H.N. Santiago
}

\begin{abstract}
Throughout this paper, our main idea is to analyze from a theoretical and normative point of view different methods to aggregate individual rankings. To do so, first we introduce the concept of a general mean on an abstract set. This new concept conciliates the Social Choice -where well-known impossibility results as the Arrovian ones are encountered- and the Decision-Making approaches -where the necessity of fusing rankings is unavoidable-. Moreover it gives rise to a reasonable definition of the concept of a ranking fusion function that does indeed satisfy the axioms of a general mean. Then we will introduce some methods to build ranking fusion functions, paying a special attention to the use of score functions, and pointing out the equivalence between ranking and scoring. To conclude, we prove that any ranking fusion function introduces a partial order on rankings implemented on a finite set of alternatives. Therefore, this allows us to compare rankings and different methods of aggregation, so that in practice one should look for the maximal elements with respect to such orders defined on rankings.
\end{abstract}

Index Terms-Ranking; Social Choice; DecisionMaking; General Means; Aggregation; Score Functions, Ranking Optimality.

\section{INTRODUCTION}

Human activities usually involve some decisionmaking procedure in order to select an alterna-

H. Bustince, M. J. Campión, J. Fernández, E. Induráin and A. Raventós-Pujol are with the Departamento de Estadística, Informática y Matemáticas. Universidad Pública de Navarra. Pamplona, Spain, (email:\{bustince,mjesus.campion,fcojavier.fernandez,steiner, armajac.raventos\}@unavarra.es)

B. Bedregal and R.H.N. Santiago are with Departamento de Informática e Matemática Aplicada - DIMAp, Universidade Federal do Rio Grande do Norte - UFRN, Natal-RN, Brazil, (e-mail: \{bedregal,regivan\}@dimap.ufrn.br)

I. Da Silva is with Diretoria de Material e Patrimônio - DMP, Universidade Federal do Rio Grande do Norte - UFRN, Natal-RN, Brazil (e-mail: ivanosca@yahoo.com.br)

H. Bustince, J. Fernandez and B. Bedregal are with the Institute of Smart Cities, Universidad Pública de Navarra, Pamplona, Spain

M. J. Campión and A. Raventós-Pujol are also with the Institute for Advanced Research in Business and Economics, Universidad Pública de Navarra, Pamplona, Spain

E. Induráin is also with InaMat ${ }^{2}$ (Institute for Advanced Materials and Mathematics, Universidad Pública de Navarra, Pamplona, Spain tive among several ones. In spite of some of the decisions being made without need of too much thought, difficult or challenging problems could demand complex solutions as well as the opinion of more than one expert, so that their evaluations should then be fused someway.

Decision-Making can be seen as the process of choosing the most appropriate one among a set of alternatives according to previously stated criteria. In general, its methods provide as a result a ranking of the alternatives. (see e.g. [3], [5], [10], [11], [12], [17], [18], [23]).

At this stage, we point out two crucial facts in this approach: On the one hand, from a theoretical point of view, the famous Arrow's impossibility theorem in mathematical Social Choice (see e.g. [2], [16]) clearly states that under an apparently mild set of common sense criteria that one may impose to a rule that fuses individual preferences into a social one, in order to retrieve or reflect in some way the main features of each of the individual components, it actually happens that the criteria are actually incompatible, so that no perfect rule is possible. On the other hand, in practical situations encountered in Decision-Making, aggregations should be done in some reasonable manner, and rankings need compulsorily be fused. This does not mean that Social Choice and Decision-Making are incompatible theories. Instead, we may consider them as complementary, so that even if we already know that no social rule will accomplish all the criteria that appear either in the Arrovian approach or in other alternative settings encountered in Social Choice ([16]), we should search for rules that satisfy other optimal properties from a more practical point of view.

It seems then crucial to conciliate the Social Choice and Decision-Making approaches by furnishing some new key concept relative to aggregation rules that, on the one hand and unlike the 
classical Social Choice settings (see [16]), lead to possibility results, and, on the other hand, it may be accepted by decision-makers as a definition of what a ranking fusion function should be.

To do so, we will work with a variation of a new axiomatic theoretical setting, recently introduced (see [7]) -in contexts of Information Sciences, Social Choice and Decision Making- to understand what a mean on an abstract set should be. Then we will introduce ranking fusion functions and score functions that do indeed satisfy the conditions involved in the axioms of that new frame.

When several methods for a decision-making problem are applied, the decision-maker will have more available information to determine a single ranking in a reasonable way. This process is usually called rank aggregation and the problem of aggregating several ranks for the same set of objects in a robust and reasonable way has a long and interesting history that goes back at least two centuries [14]. While the philosophical aspects of rank aggregation have been debated extensively, several proposals of a mathematical approach for rank aggregation have been introduced in the last years in the context of Decision-Making, see for example [1], [4], [9], [13], [19], [20]. Nevertheless, in most of them their authors do not provide minimal criteria that those rank aggregation methods should accomplish. Unlike those proposals, here we will introduce the concept of a ranking fusion function as one accomplishing that new system of axioms, formerly introduced to set well the concept of a mean in several interdisciplinary branches of Mathematics.

Obviously, different aggregation rules, even when they fit well in the new set of suitable axioms that, as mentioned above, we will introduce in the spirit of [7], could generate different rankings. So, it is reasonable to ask ourselves: Is there a ranking that could be considered optimal in some sense?

To give a reasonable answer to this last question, it is helpful to define some kind of ordering on different rankings. Thus, a ranking can be considered better than another one if it is so with respect to such ordering, once it has been implemented on the family of all possible rankings. Notice, in addition, that the fact of having an ordering defined on a set of rankings also gives us information to compare methods of aggregation or ranking fusions: given two methods of aggregation that act on the same set of rankings, we will consider the resulting outputs arising after the corresponding fusions, and then we will compare them by means of the ordering implemented. The best fused ranking as regards the ordering should be the one that we will finally select.

Bearing this in mind, in the final sections of the manuscript we will show that the ranking fusion functions that we will introduce do indeed generate a partial order on the set of all possible rankings on a finite set. This fact is crucial because it can be used, accordingly, to detect optimality among a family of rankings.

\section{OBJECTIVES AND ORGANIZATION OF THE MANUSCRIPT}

The main aims of this paper are the following:

1) Introduce a new rigorous axiomatic setting to aggregate individual preferences and rankings, as well as to formalize means on abstract sets.

2) Find a suitable notion of a ranking fusion functions, that could conciliate Social Choice and Decision-Making approaches.

3) Define the concept of a score function, and establish a key equivalence between ranking fusion functions and score functions.

4) Show that any ranking fusion function gives rise to a partial order on the family of all possible rankings, so providing us with a technique to compare different methods of aggregation of rankings, in order to detect which one is optimal.

Once the targets have been established, the present manuscript is organized accordingly, as follows:

1.- In the section III of Preliminaries, we include definitions and some previous results that are crucial for the setting and understanding of the main achievements of the manuscript. In spite of these being already known in the specialized literature, we have decided to include them here for the sake of completeness.

2.- The next section IV is crucial. There we introduce the theoretical axiomatics of a general mean on any abstract set. This key concept conciliates Social Choice and Decision-Making, so that new methods of aggregation in both disciplines should be designed now in a way that they satisfy the conditions of this new set of axioms. The system of axioms introduced 
here is new. It constitutes a slight modification of a more restrictive axiomatic system that was recently introduced in [7] in an interdisciplinary setting that includes Information Sciences.

3.- In section $\mathrm{V}$ the formal definition of a ranking fusion function is introduced along with some examples and properties. The key fact now is that ranking fusion functions do accomplish the axioms of a general mean, introduced in section IV. This gives theoretical support and consistency to our approach throughout the present manuscript.

4.- In section VI it is introduced the notion of an score function. This is also a crucial concept, because there is an equivalence between ranking fusion functions and score functions. After proving that equivalence, we may observe that score functions also fit well in that new setting of general means. Other auxiliary notions to be used in the generation of ranking fusion functions are also introduced in this section.

5.- Section VI is devoted to establish a relationship among orders on the sets of rankings and ranking fusion functions. Any ranking fusion function induces a partial order on the set of all rankings that could be defined on a finite set.. Again, this is a crucial fact, because it allows us to compare different methods of ranking fusions in order to select the optimal one, that can be determined as the method that gives rise to the best output ranking with respect to the order that had been implemented on the set of all rankings. We also provide some examples for the use of particular ranking fusion functions introduced in the previous sections. Our aim when choosing the examples introduced was just to show how to use ranking fusion functions as well as score functions. Thus, we have decided not to search for sophisticated situations, so that the examples given here may perhaps look as naive. Nevertheless, the intention behind was to use them to illustrate stepby- step how the concepts previously defined work in practice. Indeed, some of the examples furnished here has already appeared in this literature: the first one may be seen in [11] and [17], whereas the second one appears in [21]. Incidentally, we point out that the aggregating procedures used by the experts involved in both examples are quite diverse. We insist on this fact, with this examples, our aim here is to show how the processes described before work. Being our paper theoretical, so that we introduce a key new concept (namely, a general mean) and show that ranking fusion functions and score functions are general means that, in addition, define a partial order on all the rankings that could be defined on a finite set, we think that, as in any pedagogical book that establishes and develops a new concept some easy-to-understand examples should be included to highlight the key concepts introduced, for the sake of a better understanding.

6.- Finally, before the Conclusion, in section VIII, some further remarks are provided. We point out that, in the present paper, it is not our intention to introduce algorithms not to analyze a concrete -and more or less sophisticatedproblem or possible application (e.g. Information Retrieval). This will be left for further ongoing research. Here we introduce the theoretical basis and support to do so in next future.

We furnish now a "top-down" picture of the contents of the present manuscript:

A DISCUSSION ON SOCIAL CHOICE vs. DECISION MAKING: THE KEY CONCEPT OF A GENERAL MEAN

This is a new conciliating abstract concept, introduced axiomatically.

RANKING FUSION FUNCTIONS:

A key in this approach is that they are general means.

SCORE FUNCTIONS:

Construction of score functions. The concept is supported by the following crucial fact: there exists an equivalence between score functions and ranking fusion functions, as general means.

SEARCHING FOR OPTIMALITY. RANKING FUSION FUNCTIONS INDUCE PARTIAL ORDERS ON RANKINGS:

This allows us to compare different aggregation procedures looking for the best rankings, as maximal elements with respect to the partial order induced. 
To conclude this section, we give here a list of relevant mathematical symbols used throughout the paper. Despite this notation being of common use in the literature about binary relations, ordered structures or partitions of sets, we also include it for the sake of a better understanding.

$\mathcal{Q}$ : binary relation on a set; $\neg$ : negation; $\wedge$ : jointly with; $\vee$ : or; $\succsim$ : preorder on a set; $\mathcal{P}$ : profile of preferences; $\mathcal{A}$ : set of all agendas; $\geq$ : usual total order on real numbers; $\mathbb{R}$ : set of all real numbers; $\vec{A}$ : ordered partition on a set $A$ of alternatives; $\wp(A)$ : set of all ordered partitions; $\mathcal{R}$ : ranking fusion function; $\theta$ : position function; $\pi_{i}: i$-th projection function; $\vartheta_{A}$ : ordered partition generating map associated to $A$.

\section{PRELIMINARIES}

This section contains definitions and some previous results that are crucial for the setting and understanding of the main achievements of the manuscript. In spite of them being known in the specialized literature, we include them for the sake of completeness.

\section{A. Preferences}

Let $X$ denote a nonempty set.

Definition III.1. A binary relation $\mathcal{Q}$ on $X$ is a subset of the Cartesian product $X \times X$. Given two elements $x, y \in X$, we will use the standard notation $x \mathcal{Q} y$ to express that the pair $(x, y)$ belongs to $\mathcal{Q}$.

Associated to a binary relation $\mathcal{Q}$ on a set $X$, we consider its negation (respectively, its transpose) as the binary relation $\mathcal{Q}^{c}$ (respectively, $\mathcal{Q}^{t}$ ) on $X$ given by $(x, y) \in \mathcal{Q}^{c} \Leftrightarrow(x, y) \notin \mathcal{Q}$ for every $x, y \in X$ (respectively, given by $(x, y) \in \mathcal{Q}^{t} \Leftrightarrow(y, x) \in \mathcal{Q}$, for every $x, y \in X)$. We also define the adjoint $\mathcal{Q}^{a}$ of the given relation $\mathcal{Q}$, as $\mathcal{Q}^{a}=\left(\mathcal{Q}^{t}\right)^{c}$.

A binary relation $\mathcal{R}$ defined on a set $X$ is called:

(i) reflexive if $x \mathcal{Q} x$ holds for every $x \in X$,

(ii) irreflexive if $\neg(x \mathcal{Q} x)$ holds for every $x \in X$,

(iii) symmetric if $\mathcal{Q}$ and $\mathcal{Q}^{t}$ coincide,

(iv) antisymmetric if $\mathcal{Q} \cap \mathcal{Q}^{t} \subseteq\{(x, x): x \in X\}$,

(v) asymmetric if $\mathcal{Q} \cap \mathcal{Q}^{t}=\varnothing$,

(vi) total if $\mathcal{Q} \cup \mathcal{Q}^{t}=X \times X$,

(vii) transitive if $x \mathcal{Q} y \wedge y \mathcal{Q} z \Rightarrow x \mathcal{Q} z$ for every $x, y, z \in X$.

In the particular case of a nonempty set where some kind of ordering has been defined, the standard notation is different.
Definition III.2. A preorder $\succsim$ on $X$ is a binary relation on $X$ which is reflexive and transitive. An antisymmetric preorder is said to be an order, and a symmetric preorder is called an equivalence. A total preorder $\succsim$ on a set $X$ is a preorder such that if $x, y \in X$ then $(x \succsim y) \vee(y \succsim x)$ holds. If $\succsim$ is a preorder on $X$, then as usual we denote the associated asymmetric relation by $\succ$ and the associated equivalence relation by $\sim$ and these are defined by $x \succ y \Leftrightarrow(x \succsim y) \wedge \neg(y \succsim x)$ and $x \sim y \Leftrightarrow(x \succsim y) \wedge(y \succsim x)$.

A total preorder $\succsim$ defined on a nonempty set $X$ is usually called a preference on $X$.

\section{B. Arrow's impossibility results}

Suppose that $A$ is a finite set of alternatives with at least three elements. Let us assume that a finite number of $n$ agents define on $A$ their preferences. Thus, each individual will be represented by a number $i \in N_{n}=\{1, \ldots, n\}$, and her/his preference will be a total preorder $\succsim_{i}$ defined on $A$.

Definition III.3. A profile $\mathcal{P}$ of preferences is then a $n$-tuple $\left(\succsim_{1}, \ldots, \succsim_{n}\right)$ such that $\succsim_{i}$ stands for the preference of the agent $i \in N_{n}$. A nonempty subset of $A$ is said to be an agenda. If $\mathcal{A}$ denotes the set of all agendas, a choice function is a map $C: \mathcal{A} \rightarrow \mathcal{A}$ such that for every $a \in \mathcal{A}$ we have that $C(a) \subseteq a$. A social choice rule $f$ is a map that assigns a choice function $C_{\mathcal{P}}$ to each profile $\mathcal{P}$ of preferences.

Definition III.4. The Arrovian model consists of a finite set $A$ of at least three alternatives, a finite set of $n \geq 3$ agents, and a set of choice rules satisfying the following conditions:

(i) Standard domain: Each rule $f$ acts on all possible profiles of preferences on $A$, and given a profile $\mathcal{P}, f$ assigns to $\mathcal{P}$ a choice rule $C_{\mathcal{P}}$ that has in its domain all nonempty agendas.

(ii) Strong Pareto condition: For every profile $\mathcal{P}$ and every pair of alternatives $x, y \in A$ such that for every $i \in N_{n}$ we have that $x \succsim_{i} y$, and there is also at least one element $j \in N_{n}$ for which $x \succ_{j} y$, it holds true that, for every agenda $a \in \mathcal{A}$, if $x \in a$, then $y \notin C_{\mathcal{P}}(a)$.

(iii) Independence of irrelevant alternatives: For every agenda $a$ and each pair of profiles $\mathcal{P}$ and $\mathcal{P}^{\prime}$ whose restriction to the agenda $a$ coincide, the rules $C_{\mathcal{P}}$ and $C_{\mathcal{P}^{\prime}}$ that $f$ assigns to those profiles, should satisfy that $C_{\mathcal{P}}(a)=C_{\mathcal{P}^{\prime}}(a)$. 
(iv) Transitive explanation: For each profile $\mathcal{P}$ there exists a total preorder $\succsim_{\mathcal{P}, f}$ such that the corresponding choice rule $C_{\mathcal{P}}$ that $f$ assigns to $\mathcal{P}$ satisfies that $C_{\mathcal{P}}(a)=\left\{x \in a: x \succsim_{\mathcal{P}, f} y\right.$ for all $y \in a\}$, for any agenda $a$.

(v) Non-dictatorship: There is no agent $i \in N_{n}$ such that for every profile $\mathcal{P}$, every agenda $a$ and any $x, y \in A$ it holds true that $x \succsim_{i} y$ implies that if $x \in a$ then $y \notin C_{\mathcal{P}}(a)$, where $C_{\mathcal{P}}$ is the choice rule that $f$ assigns to $\mathcal{P}$.

Theorem III.5. (Arrow's impossibility theorem): The Arrovian model is empty. That is, the five conditions that appear in the model are incompatible for any rule $f$, provided that $A, N_{n}$ are finite with at least three elements each.

Remark III.6. Many different proofs of this result are encountered in the literature. It was originally published by Kenneth J. Arrow in [2]. For studies in depth on results of this kind see [16]. Once it was known that the Arrovian model leads to incompatibility, alternative approaches were introduced. But most of the new models also lead to impossibility results (see e.g Ch. 8 and ff. in [16]). However, in some models some possibility results may appear. In those models, a topology defined on the space of preferences is indeed decisive (see [8]). After that, other studies tried to keep some, but obviously not all, of the restrictions in the Arrovian model when searching for suitable aggregation rules.

\section{General MEANS}

Now we introduce the concept of a general mean on a nonempty abstract set $X$. As a matter of fact, our approach to build ranking fusion functions will be based in this key concept instead of the Arrovian approach. As aforesaid, in practice a decision-maker should compulsorily do aggregations of preferences and rankings. Thus, a clear idea about what could we understand as a mean in a totally abstract setting could be helpful for that purpose.

The task of finding a new axiomatic system to define abstract means was done in [7], looking for practical applications into multidisciplinary contexts that include Information Sciences. As a matter of fact, Definition IV.1 included below is a slight variation (indeed a bit less restrictive) that the one introduced in [7].

Thus, if we adopt now the concept of a general mean as a new setting to aggregate different in- dividual rankings, avoiding the Arrovian approach that leads to impossibility results, the output ranking resulting of the computation of corresponding mean will indeed act as a social ranking. The good news is that general means do actually exist in most contexts, as analyzed in [7].

Definition IV.1. A sequence of $X$-valued maps $\left(M_{n}\right)_{n=1}^{\infty}$, where each $M_{n}$ is defined on the Cartesian product $X^{n}$, is said to be a general mean on $X$ if it satisfies the following axioms:

- GM1 (Anonymity-neutrality) For every $n \in \mathbb{N}$ and $\left(x_{1}, \ldots, x_{n}\right) \in X^{n}$ it holds true that $M_{n}\left(x_{1}, \ldots, x_{n}\right)=M_{n}\left(x_{\sigma(1)}, \ldots, x_{\sigma(n)}\right)$ for any permutation $\sigma$ of the set $\{1, \ldots, n\}$.

- GM2 (Unanimity) For any $n \in \mathbb{N}$ and $x \in X$ it holds true that $M_{n}(x, \ldots(n$ times $) \ldots, x)=x$.

- GM3 (Stability) Given $n \in \mathbb{N}$ and $\left(x_{1}, \ldots, x_{n}\right) \in X^{n}$, it holds that $M_{n+1}\left(x_{1}\right.$, $\left.\ldots, x_{n}, M_{n}\left(x_{1}, \ldots, x_{n}\right)\right)=M_{n}\left(x_{1}, \ldots, x_{n}\right)$.

If $\left(M_{n}\right)_{n=1}^{\infty}$ is a general mean on $X$, given $k$ elements $\left\{x_{1}, \ldots, x_{k}\right\}$, the element $\bar{x}=$ $M_{k}\left(x_{1}, \ldots, x_{k}\right) \in X$ is usually called the mean of the elements $x_{i}(i=1, \ldots, k)$.

\section{RANKING FUSION FUNCTIONS}

The main concept of a ranking fusion function is introduced now (see Definition V.3 below) as a particular kind of a mean, in the new setting just introduced above in Definition IV.1. This concept can be considered as a bridge where Social Choice and Decision-Making meet, so that it conciliates both approaches.

Definition V.1. Again we denote by $N_{n}$ the set $\{1, \ldots, n\}$ for each natural number $n \in \mathbb{N}$.

Given a finite and nonempty set of alternatives $A=\left\{a_{1}, \ldots, a_{n}\right\}$, define $\wp_{A}=\left\{\left(A_{1}, \ldots, A_{k}\right)\right.$ : $\left\{A_{1}, \ldots, A_{k}\right\}$ is a partition of $\left.A\right\}$. Henceforward, each element of $\wp_{A}$ is said to be an ordered partition of the set of alternatives $A$. So $\wp_{A}$ is the family of all ordered partitions of the set of alternatives $A$.

Definition V.2. Given an ordered partition $\vec{A}=$ $\left(A_{1}, \ldots, A_{k}\right) \in \wp_{A}$, with $A_{i}=\left\{a_{i 1}, \ldots, a_{i p_{i}}\right\}$, we will associate to it, in a natural way, the following total preorder $\succsim_{\vec{A}}$ or ranking on the set of alternatives $A$, given as follows: $a_{11} \sim_{\vec{A}} \ldots \sim_{\vec{A}} a_{1 p_{1}} \succ_{\vec{A}}$ $a_{21} \sim_{\vec{A}} \ldots \sim_{\vec{A}} a_{2 p_{2}} \succ_{\vec{A}} \ldots \succ_{\vec{A}} a_{k 1} \sim_{\vec{A}} \ldots \sim_{\vec{A}} a_{k p_{k}}$ meaning that the alternative $a_{i j}$ and $a_{i(j+1)}$ are equivalent, i.e. it is not possible to discern among 
TABLE I

EXAMPLE OF SEVEN RANKINGS FOR SIX ALTERNATIVES.

\begin{tabular}{|c|c|}
\hline & Ranking \\
\hline$\vec{A}_{1}$ & $a_{2}>a_{4} \sim a_{5}>a_{1}>a_{3}>a_{6}$ \\
$\vec{A}_{2}$ & $a_{3} \sim a_{5}>a_{1}>a_{4}>a_{2} \sim a_{6}$ \\
$\vec{A}_{3}$ & $a_{5}>a_{2}>a_{3}>a_{4}>a_{1}>a_{6}$ \\
$\vec{A}_{4}$ & $a_{5}>a_{3} \sim a_{2}>a_{4}>a_{6}>a_{1}$ \\
$\vec{A}_{5}$ & $a_{5} \sim a_{2}>a_{1}>a_{4}>a_{3}>a_{6}$ \\
$\vec{A}_{6}$ & $a_{3}>a_{2} \sim a_{1}>a_{4} \sim a_{6}>a_{5}$ \\
$\vec{A}_{7}$ & $a_{5}>a_{3}>a_{2} \sim a_{4}>a_{1}>a_{6}$ \\
\hline
\end{tabular}

the two alternatives, and $a_{m n}$ is better than the alternative $a_{(m+1) l}$ for each $i \in N_{k}, j \in N_{p_{i}-1}$, $m \in N_{k-1}, n \in N_{p_{m}}$ and $l \in N_{p_{m+1}}$. For example, $\left(\left\{a_{1}, a_{2}\right\},\left\{a_{3}\right\},\left\{a_{4}, a_{5}, a_{6}\right\}\right)$ denoted the ranking $a_{1} \sim_{\vec{A}} a_{2} \succ_{\vec{A}} a_{3} \succ_{\vec{A}} a_{4} \sim_{\vec{A}} a_{5} \sim_{\vec{A}} a_{6}$.

The total preorder $\succsim_{\vec{A}}$ is said to be the natural preference associated to the ordered partition $\vec{A}$.

Definition V.3. Let $A$ be a finite set of alternatives. A ranking fusion function (RFF) for $A$ is any general mean defined on the family $\wp_{A}$ of all the ordered partitions of the set $A$. Equivalently, it is a general mean defined on the set of all the total preorders that may be defined on $A$.

Example V.4. Let $\vec{A}_{i}=\left(A_{i 1}, \ldots, A_{i k_{i}}\right) \in \wp_{A}$ for $i \in N_{m}$ and $A=\left\{a_{1}, \ldots, a_{n}\right\}$. Define

- $A_{1}^{\prime}=\bigcup_{i=1}^{m} A_{i 1}$ and

- $A_{j}^{\prime}=\bigcup_{i=1}^{m} A_{i j}-\bigcup_{l=1}^{j-1} A_{l}^{\prime}$ for each $j=2, \ldots, n$

with $A_{i j}=\emptyset$ provided that $j>k_{i}$.

Let $\mathcal{R}_{0}: \bigcup_{m=1}^{\infty} \wp_{A}^{m} \rightarrow \wp_{A}$ be the map defined by $\mathcal{R}_{0}\left(\vec{A}_{1}^{m}, \ldots, \vec{A}_{m}\right)=\left(A_{1}, \ldots, A_{k}\right)$ where $\left(A_{1}, \ldots, A_{k}\right)$ comes from $\left(A_{1}^{\prime}, \ldots, A_{m}^{\prime}\right)$ after removing the $A_{j}^{\prime}$ which are empty. Notice here that always $A_{1}^{\prime} \neq \emptyset$. Formally, for every $j \in N_{k}$ we have that $A_{j}=A_{i}^{\prime}$, where $i=\min \left\{l: A_{l}^{\prime} \neq \emptyset\right.$ and $A_{l}^{\prime} \neq A_{h}$ for each $\left.h<j\right\}$. It is straightforward to see that $\mathcal{R}_{0}$ is a ranking fusion function.

Now, we will apply this ranking fusion function to the rankings of the alternatives $A=$ $\left\{a_{1}, \ldots, a_{6}\right\}$ shown in the Table I. The Table II shows the subsets $A_{j}^{\prime}$ with $j \in N_{6}$ and $A_{j}$ with $j=1, \ldots, k=3$. Therefore, $\mathcal{R}_{0}\left(\vec{A}_{1}, \ldots, \vec{A}_{7}\right)=$ $\left(\left\{a_{2}, a_{3}, a_{5}\right\},\left\{a_{1}, a_{4}\right\},\left\{a_{6}\right\}\right)$, i.e. the fusion of these seven rankings is $a_{2} \sim a_{3} \sim a_{5}>a_{1} \sim a_{4}>a_{6}$.

Proposition V.5. Let $\mathcal{R}: \bigcup_{j=1}^{\infty} \wp_{A}^{j} \rightarrow \wp_{A}$ be a ranking fusion function and $\gamma: \wp_{A} \rightarrow \wp_{A}$ be a bijection.
TABLE II

FUSION OF THE SEVEN RANKINGS.

\begin{tabular}{|l|c|c|c|c|c|c|}
\hline $\mathrm{j}$ & 1 & 2 & 3 & 4 & 5 & 6 \\
\hline$A_{j}^{\prime}$ & $\left\{a_{2}, a_{3}, a_{5}\right\}$ & $\left\{a_{1}, a_{4}\right\}$ & $\emptyset$ & $\left\{a_{6}\right\}$ & $\emptyset$ & $\emptyset$ \\
$A_{j}$ & $\left\{a_{2}, a_{3}, a_{5}\right\}$ & $\left\{a_{1}, a_{4}\right\}$ & $\left\{a_{6}\right\}$ & & & \\
\hline
\end{tabular}

Then the function $\mathcal{R}_{\gamma}: \bigcup_{j=1}^{\infty} \wp_{A}^{j} \rightarrow \wp_{A}$ defined by $\mathcal{R}_{\gamma}\left(\overrightarrow{A_{1}}, \ldots, \overrightarrow{A_{m}}\right)=\gamma^{-1}\left(\mathcal{R}\left(\gamma\left(\overrightarrow{A_{1}}\right), \ldots, \gamma\left(\overrightarrow{A_{m}}\right)\right)\right)$ is also a ranking fusion function, called the $\gamma-$ conjugate of $\mathcal{R}$.

Proof. It is plain that if $\mathcal{R}$ satisfies GM1 and GM2, so does $\mathcal{R}_{\gamma}$ too. On the other hand, with respect to GM3 we have that: $\quad \mathcal{R}_{\gamma}\left(\overrightarrow{A_{1}}, \ldots, \overrightarrow{A_{m}}, \mathcal{R}_{\gamma}\left(\vec{A}_{1}, \ldots, \vec{A}_{m}\right)\right)=$ $\mathcal{R}_{\gamma}\left(\overrightarrow{A_{1}}, \ldots, \vec{A}_{m}, \gamma^{-1}\left(\mathcal{R}\left(\gamma\left(\overrightarrow{A_{1}}\right), \ldots, \gamma\left(\overrightarrow{A_{m}}\right)\right)\right)\right)=$ $\gamma^{-1}\left(\mathcal{R}\left(\gamma\left(\overrightarrow{A_{1}}\right), \ldots, \quad \gamma\left(\overrightarrow{A_{m}}\right), \gamma\left(\gamma^{-1}\left(\mathcal{R}\left(\gamma\left(\overrightarrow{A_{1}}\right), \ldots\right.\right.\right.\right.\right.$, $\left.\left.\left.\left.\gamma\left(\vec{A}_{m}\right)\right)\right)\right)\right)=\gamma^{-1}\left(\mathcal{R}\left(\gamma\left(\vec{A}_{1}\right), \ldots, \gamma\left(\vec{A}_{m}\right), \mathcal{R}\left(\gamma\left(\overrightarrow{A_{1}}\right)\right.\right.\right.$, $\left.\left.\left.\ldots, \gamma\left(\vec{A}_{m}\right)\right)\right)\right)=\gamma^{-1}\left(\mathcal{R}\left(\gamma\left(\vec{A}_{1}\right), \ldots, \gamma\left(\vec{A}_{m}\right)\right)\right)=$ $\mathcal{R}_{\gamma}\left(\vec{A}_{1}, \ldots, \vec{A}_{m}\right)$. Therefore, $\mathcal{R}_{\gamma}$ also satisfies GM3. Hence, it is a ranking fusion function.

Definition V.6. Given a set $A$ of alternatives, a ranking fusion function $\mathcal{R}: \bigcup_{j=1}^{\infty} \wp_{A}^{j} \rightarrow \wp_{A}$ such that for each $\vec{A}_{1}, \ldots, \vec{A}_{m} \in \wp_{A}$ it holds true that: $\mathcal{R}\left(\overrightarrow{A_{1}}, \ldots, \overrightarrow{A_{m}}\right)=\mathcal{R}\left(\vec{A}_{1}, \ldots, \overrightarrow{A_{m}}, \overrightarrow{A_{1}}\right)$, is said to be unaffected by duplications of ranking.

Remark V.7. In many typical situations it is interesting that repeated rankings do not affect to the final output ranking got after the aggregation. A trivial example appears when the rankings are already ordered, and we would like to select a maximal one as regards that given order. By this reason we analyze now when a ranking fusion function is not affected by repetitions of rankings.

In the abstract framework of abstract means defined on nonempty set, other properties of this kind have already been studied in depth in Section 4 of [7], under the name of iterativity properties.

Proposition V.8. Let $\mathcal{R}: \bigcup_{j=1}^{\infty} \wp_{A}^{j} \rightarrow \wp_{A}$ be $a$ ranking fusion function. Then $\mathcal{R}^{\prime}: \bigcup_{j=1}^{\infty} \wp_{A}^{j} \rightarrow \wp_{A}$ defined by $\mathcal{R}^{\prime}\left(\vec{A}_{1}, \ldots, \vec{A}_{m}\right)=\mathcal{R}\left({\overrightarrow{A^{\prime}}}_{1}, \ldots, \vec{A}^{\prime}{ }_{k}\right)$, with $\left\{{\overrightarrow{A^{\prime}}}_{1}, \ldots,{\overrightarrow{A^{\prime}}}_{k}\right\}=\left\{\overrightarrow{A_{1}}, \ldots, \overrightarrow{A_{m}}\right\}$, is a RFF that is unaffected by duplications of ranking.

Proof. Clearly, since $\mathcal{R}$ satisfies GM1, and the fact 
that the removal of copies of some ranking does not have consequences on that condition, the new fusion function $\mathcal{R}^{\prime}$ also accomplishes GM1. Let $\vec{A} \in$ $\wp_{A}$. Then $\mathcal{R}^{\prime}(\vec{A}, \ldots(n$ times $) \ldots, \vec{A})=\mathcal{R}(\vec{A})=\vec{A}$ and therefore $\mathcal{R}^{\prime}$ also satisfies GM2. In addition, as regards GM3, let $\left\{{\overrightarrow{A^{\prime}}}_{1}, \ldots,{\overrightarrow{A^{\prime}}}_{k}\right\}=\left\{\overrightarrow{A_{1}}, \ldots, \overrightarrow{A_{m}}\right\}$.

Case 1: If $\mathcal{R}^{\prime}\left(\vec{A}_{1}, \ldots, \overrightarrow{A_{m}}\right) \in\left\{{\overrightarrow{A^{\prime}}}_{1}, \ldots,{\overrightarrow{A^{\prime}}}_{k}\right\}$ then we have that: $\mathcal{R}^{\prime}\left(\vec{A}_{1}, \ldots, \overrightarrow{A_{m}}, \mathcal{R}^{\prime}\left(\vec{A}_{1}, \ldots, \overrightarrow{A_{m}}\right)\right)=$ $\mathcal{R}\left({\overrightarrow{A^{\prime}}}_{1}, \ldots, \overrightarrow{A^{\prime}}{ }_{k}\right)=\mathcal{R}^{\prime}\left(\overrightarrow{A_{1}}, \ldots, \overrightarrow{A_{m}}\right)$.

Case 2: If $\mathcal{R}^{\prime}\left(\vec{A}_{1}, \ldots, \overrightarrow{A_{m}}\right) \notin\left\{{\overrightarrow{A^{\prime}}}_{1}, \ldots,{\overrightarrow{A^{\prime}}}_{k}\right\}$ then it follows that: $\mathcal{R}^{\prime}\left(\vec{A}_{1}, \ldots, \overrightarrow{A_{m}}, \mathcal{R}^{\prime}\left(\vec{A}_{1}, \ldots, \overrightarrow{A_{m}}\right)\right)=$ $\mathcal{R}\left({\overrightarrow{A^{\prime}}}_{1}, \ldots,{\overrightarrow{A^{\prime}}}_{k}, \mathcal{R}^{\prime}\left(\overrightarrow{A_{1}}, \ldots, \overrightarrow{A_{m}}\right)\right)=\mathcal{R}\left({\overrightarrow{A^{\prime}}}_{1}\right.$, $\left.\ldots,{\overrightarrow{A^{\prime}}}_{k}, \mathcal{R}\left({\overrightarrow{A^{\prime}}}_{1}, \ldots,{\overrightarrow{A^{\prime}}}_{k}\right)\right)=\mathcal{R}\left({\overrightarrow{A^{\prime}}}_{1}, \ldots,{\overrightarrow{A^{\prime}}}_{k}\right)=$ $\mathcal{R}^{\prime}\left(\vec{A}_{1}, \ldots, \vec{A}_{m}\right)$. Therefore, $\mathcal{R}$ satisfies GM3 and, consequently, it is a ranking fusion function which is clearly unaffected by duplications of ranking.

\section{SCORE FUNCTIONS}

In the present section we will formally introduce the concept of an $A$-score function on a finite set of alternatives $A$, taking into account its corresponding set $\wp_{A}$ of ordered partitions.

The main definition to be introduced here (namely Definition VI.3 below) is not whimsical. It seeks to have an abstract frame of reference, and a way of acting that respects the previous context of general means and ranking fusion functions. By this reason, we will define this concept through a new system of axioms. Then we will prove a key equivalence between ranking fusion functions and score functions.

To start with, first we include some auxiliary previous definitions.

Definition VI.1. Let $A$ stand for a finite set of alternatives whose cardinality is $n$. The function $\theta: A \times \wp_{A} \rightarrow N_{n}$ defined, for each $a \in A$ and $\vec{A}=\left(A_{1}, \ldots, A_{k}\right) \in \wp_{A}$, as follows:

$\theta(a, \vec{A})= \begin{cases}1 & \text { if } a \in A_{1} \\ 1+\operatorname{Card}\left(\bigcup_{p=1}^{l-1} A_{p}\right) & \text { if } a \in A_{l} \text { for } l \geq 2\end{cases}$

is said to be a position function .

For example, working with the set $A=$ $\left\{a_{1}, \ldots, a_{6}\right\}$ and the ordered partition $\vec{A}=$ ( $\left.\left\{a_{1}\right\},\left\{a_{3}, a_{4}\right\},\left\{a_{2}, a_{5}\right\},\left\{a_{6}\right\}\right)$ we have that:

$\theta\left(a_{1},\left(\left\{a_{1}\right\},\left\{a_{3}, a_{4}\right\},\left\{a_{2}, a_{5}\right\},\left\{a_{6}\right\}\right)\right)=1$,

$\theta\left(a_{2},\left(\left\{a_{1}\right\},\left\{a_{3}, a_{4}\right\},\left\{a_{2}, a_{5}\right\},\left\{a_{6}\right\}\right)\right)=1+$ $\operatorname{Card}\left(\left\{a_{1}, a_{3}, a_{4}\right\}\right)=4$,

$\begin{array}{lll}\theta\left(a_{3},\left(\left\{a_{1}\right\},\left\{a_{3}, a_{4}\right\},\left\{a_{2}, a_{5}\right\},\left\{a_{6}\right\}\right)\right) & = & 1+ \\ \operatorname{Card}\left(\left\{a_{1}\right\}\right)=2, & & \\ \quad \theta\left(a_{4},\left(\left\{a_{1}\right\},\left\{a_{3}, a_{4}\right\},\left\{a_{2}, a_{5}\right\},\left\{a_{6}\right\}\right)\right) & = & 1+ \\ \operatorname{Card}\left(\left\{a_{1}\right\}\right)=2, & & \\ \quad \theta\left(a_{5},\left(\left\{a_{1}\right\},\left\{a_{3}, a_{4}\right\},\left\{a_{2}, a_{5}\right\},\left\{a_{6}\right\}\right)\right) & = & 1+ \\ \operatorname{Card}\left(\left\{a_{1}, a_{3}, a_{4}\right\}\right)=4, & & \\ \quad \theta\left(a_{6},\left(\left\{a_{1}\right\},\left\{a_{3}, a_{4}\right\},\left\{a_{2}, a_{5}\right\},\left\{a_{6}\right\}\right)\right) & = & 1+\end{array}$
$\operatorname{Card}\left(\left\{a_{1}, a_{3}, a_{4}, a_{2}, a_{5}\right\}\right)=6$.

Definition VI.2. Let $A$ be a set of alternatives, $n=\operatorname{Card}(A)$ and $\rho_{A}: N_{n} \rightarrow A$ be the bijection given by $\rho_{A}(i)=a_{i}$. The function $\vartheta_{A}:\left(N_{n}\right)^{n} \rightarrow \wp_{A}$ defined by $\vartheta_{A}\left(k_{1}, \ldots, k_{n}\right)=\left\{\left(A_{1}\right)\right.$ if $k_{i}=k_{j}$ for each $i, j \in$ $N_{n}$ and $\left(A_{1}, \vartheta_{A^{\prime}}\left(k_{1}^{\prime}, \ldots, k_{p}^{\prime}\right)\right)$ otherwise $\}$, where $A_{1}=\left\{\rho_{A}(i): k_{i}=\max \left(k_{1}, \ldots, k_{n}\right)\right\}, A^{\prime}=A \backslash A_{1}$, $p=n-\operatorname{Card}\left(A_{1}\right),\left(k_{1}^{\prime}, \ldots, k_{p}^{\prime}\right)$ is $\left(k_{1}, \ldots, k_{n}\right)$ after removing the $k_{i}$ 's such that $\rho_{A}(i) \in A_{1}$, and obviously $\vartheta_{A^{\prime}}\left(k_{1}^{\prime}, \ldots, k_{p}^{\prime}\right)$ is recursively got as we did with $\vartheta_{A}\left(k_{1}, \ldots, k_{n}\right)$, if necessary defining new sets $A^{\prime \prime}=A^{\prime} \backslash A_{1}^{\prime}$, etc., is called the ordered partition generating map associated to $A$.

For example, $\vartheta_{A}(2,5,2,4,1,5)$ $\left(\left\{a_{2}, a_{6}\right\},\left\{a_{4}\right\},\left\{a_{1}, a_{3}\right\},\left\{a_{5}\right\}\right)$.

Notice that if we start with an ordered partition $\vec{A}$ of the set $A$, and we consider now the $n$-tuple $\left(\theta\left(a_{1}, \vec{A}\right), \ldots, \theta\left(a_{n}, \vec{A}\right)\right) \in\left(N_{n}\right)^{n}$, then $\vartheta_{A}\left(\theta\left(a_{1}, \vec{A}\right), \ldots, \theta\left(a_{n}, \vec{A}\right)\right)$ retrieves $\vec{A}$ but ordered in the opposite sense.

Henceforward $\pi_{i}$ will denote the $i-t h$ projection function defined on the domain $\mathbb{N}^{n}, 1 \leq i \leq n$, where $\mathbb{N}$ stands for the set of natural numbers.

Definition VI.3. Let $A$ be a set of $n$ alternatives. A function $M: \bigcup_{m=1}^{\infty} \wp_{A}^{m} \rightarrow \mathbb{N}^{n}$ is said to be an $A$ score function if for each strictly positive $m \in \mathbb{N}$ it accomplishes the following conditions:

- SF1 The restriction of $M$ to $\wp_{A}^{m}$ is commutative, that is $M\left(\vec{A}_{1}, \ldots, \vec{A}_{m}\right)=$ $M\left(\vec{A}_{\sigma(1)}, \ldots, \vec{A}_{\sigma(m)}\right)$ for any permutation $\sigma$ of the set $N_{m}=\{1, \ldots, m\}$.

- SF2 Let $j, l \in N_{n}$. If $\theta\left(a_{j}, \overrightarrow{A_{i}}\right) \leq \theta\left(a_{l}, \vec{A}_{i}\right)$ for each $i \in N_{m}$ then $\pi_{j}\left(M\left(\overrightarrow{A_{1}}, \ldots, \overrightarrow{A_{m}}\right)\right) \geq$ $\pi_{l}\left(M\left(\vec{A}_{1}, \ldots, \vec{A}_{m}\right)\right)$.

- SF3 $\vartheta_{A}\left(M\left(\overrightarrow{A_{1}}, \ldots, \vec{A}_{m}, \vartheta_{A}\left(M\left(\vec{A}_{1}, \ldots\right.\right.\right.\right.$, $\left.\left.\left.\vec{A}_{m}\right)\right)\right)=\vartheta_{A}\left(M\left(\vec{A}_{1}, \ldots, \vec{A}_{m}\right)\right)$.

- SF4 $\pi_{i}\left(M\left(\overrightarrow{A_{1}}, \ldots, \overrightarrow{A_{m}}\right)\right)$ $\pi_{i}\left(M\left(\vec{A}_{1}, \ldots, \vec{A}_{m+1}\right)\right)$ for each $i \in N_{n}$. 
Proposition VI.4. Let $A$ be a set of $n$ alternatives and $M$ an A-score function. The following properties hold true:

- If $\theta\left(a, \vec{A}_{i}\right)=1$ for each $i \in N_{m}$ then $\theta\left(a, \vartheta_{A}\left(M\left(\vec{A}_{1}, \ldots, \overrightarrow{A_{m}}\right)\right)\right)=1$.

- If $\theta\left(a, \vec{A}_{i}\right)=n$ for each $i \in N_{m}$ then $\theta\left(a, \vartheta_{A}\left(M\left(\overrightarrow{A_{1}}, \ldots, \overrightarrow{A_{m}}\right)\right)\right)=n$.

- If $\vec{A}_{i}=\vec{A}_{j}$ for each $i, j \in N_{m}$ then $\theta\left(a, \vartheta_{A}\left(M\left(\vec{A}_{1}, \ldots, \vec{A}_{m}\right)\right)\right)=\theta\left(a, \vec{A}_{1}\right)$ for any $a \in A$.

Proof. All this is immediate.

Example VI.5. Let $A=\left\{a_{1}, \ldots, a_{n}\right\}$. We introduce now two ranking fusion functions on a finite set $A$ of $n$ alternatives. Both of them will be used henceforward, and are based on the concept of an $A$-score function just launched in Definition VI.3.

1.- Let $M_{1}: \wp_{A}^{m} \rightarrow \mathbb{N}^{n}$ be defined by $M_{1}\left(\overrightarrow{A_{1}}, \ldots, \overrightarrow{A_{m}}\right)=\left(b_{1}, \ldots, b_{n}\right)$ with $b_{j}=$ $m n-\sum_{i=1}^{m} \theta\left(a_{j}, \vec{A}_{i}\right), \quad$ for each $j \in N_{n}=$ $\{1, \ldots, n\}$. Define $\mathcal{R}_{1}: \bigcup_{m=1}^{\infty} \wp_{A}^{m} \rightarrow \wp_{A}$ by $\mathcal{R}_{1}\left(\vec{A}_{1}, \ldots, \overrightarrow{A_{m}}\right)=\left(A_{1}, \ldots, A_{k}\right)$ such that:

(i) $A_{1}=\left\{a_{j} \in A: b_{j}=\max \left(b_{1}, \ldots, b_{n}\right)\right\}$,

(ii) for every $i=2, \ldots, k, A_{i}=\left\{a_{j} \in A\right.$ : $a_{j} \notin \bigcup_{h<i} A_{h}$ and $b_{j} \geq b_{l}$ for each $l$ such that $\left.a_{l} \notin \bigcup_{h<i} A_{h}\right\}$.

(iii) $\left(A_{1}, \ldots, A_{k}\right) \in \wp_{A}$.

Then $\mathcal{R}_{1}$ is a ranking fusion function.

2.- Let $M_{2}: \bigcup_{m=1}^{\infty} \wp_{A}^{m} \rightarrow \mathbb{N}^{n}$ be defined by $M_{2}\left(\vec{A}_{1}, \ldots, \overrightarrow{\vec{A}_{m}=1}\right)=\left(b_{1}, \ldots, b_{n}\right)$, with $b_{j}=$ $\sum_{l=1}^{n} \sum_{i=1}^{m} p\left(\theta\left(a_{j}, \vec{A}_{i}\right), \theta\left(a_{l}, \vec{A}_{i}\right)\right)$ for each $j \in N_{n}$, where $p: N_{n} \times N_{n} \rightarrow\{0,1\}$ is given by

$$
p(j, l)= \begin{cases}1 & \text { if } j<l \\ 0 & \text { otherwise }\end{cases}
$$

Define $\mathcal{R}_{2}: \bigcup_{m=1}^{\infty} \wp_{A}^{m} \rightarrow \wp_{A}$ by declaring that $\mathcal{R}_{2}\left(\vec{A}_{1}, \ldots, \overrightarrow{\vec{A}_{m}}\right)=\left(A_{1}, \ldots, A_{k}\right)$ such that:

(i) $A_{1}=\left\{a_{j} \in A: b_{j}=\max \left(b_{1}, \ldots, b_{n}\right)\right\}$,

(ii) for each $i=2, \ldots, k, A_{i}=\left\{a_{j} \in A\right.$ : $b_{j} \notin \bigcup_{h<i} A_{h}$ and $b_{j} \geq b_{l}$ for each $l$ such that $\left.a_{l} \notin \bigcup_{h<i} A_{h}\right\}$.

(iii) $\left(A_{1}, \ldots, A_{k}\right) \in \wp_{A}$.
Then $\mathcal{R}_{2}$ is a ranking fusion function.

Thus, if we have at hand the following three rankings:

$$
\begin{aligned}
& \overrightarrow{A_{1}}=\left(\left\{a_{1}\right\},\left\{a_{2}\right\},\left\{a_{3}\right\},\left\{a_{4}\right\}\right) \text { i.e. } a_{1}>a_{2}> \\
& a_{3}>a_{4}, \\
& \overrightarrow{A_{2}}=\left(\left\{a_{2}\right\},\left\{a_{1}\right\},\left\{a_{3}, a_{4}\right\}\right), \text { i.e. } a_{2}>a_{1}> \\
& a_{3} \sim a_{4} \text {, and } \\
& \overrightarrow{A_{3}}=\left(\left\{a_{1}\right\},\left\{a_{2}, a_{3}\right\},\left\{a_{4}\right\}\right), \text { i.e. } a_{1}>a_{2} \sim \\
& a_{3}>a_{4},
\end{aligned}
$$

then we get $M_{1}\left(\overrightarrow{A_{1}}, \overrightarrow{A_{2}}, \overrightarrow{A_{3}}\right)=(12-(1+2+$ 1), $12-(2+1+2), 12-(3+3+2), 12-(4+$ $3+4))=(8,7,4,1)$. Therefore $\mathcal{R}_{1}\left(\overrightarrow{A_{1}}, \overrightarrow{A_{2}}, \overrightarrow{A_{3}}\right)=$ $\left(\left\{a_{1}\right\},\left\{a_{2}\right\},\left\{a_{3}\right\},\left\{a_{4}\right\}\right)$. In other words, here we have that $a_{1}>a_{2}>a_{3}>a_{4}$. Similarly $\mathcal{R}_{2}\left(\overrightarrow{A_{1}}, \overrightarrow{A_{2}}, \overrightarrow{A_{3}}\right)=\left(\left\{a_{1}\right\},\left\{a_{2}\right\},\left\{a_{3}\right\},\left\{a_{4}\right\}\right)$.

Also, if we consider now the following three rankings:

$$
\begin{aligned}
& \overrightarrow{A_{1}}=\left(\left\{a_{1}\right\},\left\{a_{2}\right\},\left\{a_{3}\right\},\left\{a_{4}\right\}\right) \text { i.e. } a_{1}>a_{2}> \\
& a_{3}>a_{4}, \\
& \overrightarrow{A_{2}}=\left(\left\{a_{4}\right\},\left\{a_{1}\right\},\left\{a_{2}\right\},\left\{a_{3}\right\}\right) \text { i.e. } a_{4}>a_{1}> \\
& a_{2}>a_{3}, \text { and } \\
& \overrightarrow{A_{3}}=\left(\left\{a_{2}\right\},\left\{a_{3}\right\},\left\{a_{1}\right\},\left\{a_{4}\right\}\right) \text { i.e. } a_{2}>a_{3}> \\
& a_{1}>a_{4},
\end{aligned}
$$

then we obtain $M_{1}\left(\overrightarrow{A_{1}}, \overrightarrow{A_{2}}, \overrightarrow{A_{3}}\right)=(12-(1+2+$ $3), 12-(2+3+1), 12-(3+4+2), 12-(4+1+4))=$ $(6,6,3,3)$. In the same way, $M_{2}\left(\overrightarrow{A_{1}}, \overrightarrow{A_{2}}, \overrightarrow{A_{3}}\right)=$ $(0+2+2+2,1+0+3+2,1+0+0+2,1+$ $1+1+0)=(6,6,3,3)$. Hence $\mathcal{R}_{1}\left(\vec{A}_{1}, \vec{A}_{2}, \vec{A}_{3}\right)=$ $\mathcal{R}_{2}\left(\overrightarrow{A_{1}}, \overrightarrow{A_{2}}, \overrightarrow{A_{3}}\right)=\left(\left\{a_{1}, a_{2}\right\},\left\{a_{3}, a_{4}\right\}\right)$. In other words both fusion ranking functions give rise to the ranking: $a_{1} \sim a_{2}>a_{3} \sim a_{4}$.

In both examples, the function $M_{i}$ (with $i \in$ $\{1,2\}$ ) plays a fundamental role in order to obtain the ranking fusion function $\mathcal{R}_{i}$. The following theorem generalizes and formalizes this process.

This Theorem VI.6 is essential to our approach, since it establishes the relationship -in fact an equivalence once Theorem VI.9 below is provedbetween $A$-score functions and ranking fusion functions.

Theorem VI.6. Let $A={ }_{\infty}\left\{a_{1}, \ldots, a_{n}\right\}$. For each A-score function $M: \bigcup_{m=1}^{\infty} \wp_{A}^{m} \rightarrow \mathbb{N}^{n}$ it holds true that the function $\mathcal{R}_{M}: \bigcup_{m=1}^{\infty} \wp_{A}^{m} \rightarrow \wp_{A}$, given by $\mathcal{R}_{M}\left(\vec{A}_{1}, \ldots, \vec{A}_{m}\right)=\vartheta_{A}\left(M\left(\vec{A}_{1}, \ldots, \vec{A}_{m}\right)\right)$ is a ranking fusion function. 
Proof. The anonymity-neutrality condition GM1 for $\mathcal{R}_{M}$ follows from the commutativity of $M$. We will prove now the unanimity condition GM2 for $\mathcal{R}_{M}$, by induction on $m$. For $m=1$ we may notice that by the condition $\mathrm{SF} 2$ and the definition of $\vartheta_{A}$ it follows that $\vartheta_{A}(M(\vec{A}))=\vec{A}$ and therefore $\mathcal{R}_{M}(\vec{A})=\vartheta_{A}(M(\vec{A}))=\vec{A}$. If we use the inductive hypothesis (IH) $\mathcal{R}_{M}(\underbrace{\vec{A}, \ldots, \vec{A}}_{m \text {-times }})=\vec{A}$, then we get that: $\mathcal{R}_{M}(\underbrace{\vec{A}, \ldots, \vec{A}}_{(m+1) \text {-times }})=\vartheta_{A}(M(\underbrace{\vec{A}, \ldots, \vec{A}}_{(m+1) \text {-times }}))$ (by definition $)=\vartheta_{A}\left(M(\underbrace{\vec{A}, \ldots, \vec{A}}_{m \text {-times }}, \vartheta_{A}(M(\underbrace{\vec{A}, \ldots, \vec{A}}_{m \text {-times }})))\right.$ (by $\mathrm{IH})=\vartheta_{A}(M(\underbrace{\vec{A}, \ldots, \vec{A}}_{m-\text { times }}))$ (by SF3) $=\vec{A}$, again by IH. Finally, the weak compatibility condition GM3 is straightforwardly got from the definition of $\mathcal{R}_{M}$ and SF3.

Now we introduce a preparatory lemma, in order to prove the aforementioned equivalence between ranking fusion functions and score functions.

Lemma VI.7. Let $A=\left\{a_{1}, \ldots, a_{n}\right\}$ be $a$ set of alternatives. For each ranking fusion function $\mathcal{R}: \bigcup_{m=1}^{\infty} \wp_{A}^{m} \rightarrow \wp_{A}$ the function $M_{\mathcal{R}}: \bigcup_{m=1}^{\infty} \wp_{A}^{m} \rightarrow N^{n}$ given by $M_{\mathcal{R}}\left(\vec{A}_{1}, \ldots, \overrightarrow{A_{m}}\right)=$ $\left[m(n+1)-\theta\left(a_{1}, \mathcal{R}\left(\overrightarrow{A_{1}}, \ldots, \overrightarrow{A_{m}}\right)\right), \ldots\right.$, $\left.m(n+1)-\theta\left(a_{n}, \mathcal{R}\left(\vec{A}_{1}, \ldots, \vec{A}_{m}\right)\right)\right]$ is such that $\vartheta_{A}\left(M_{\mathcal{R}}\left(\overrightarrow{A_{1}}, \ldots, \overrightarrow{A_{m}}\right)\right)=\mathcal{R}\left(\overrightarrow{A_{1}}, \ldots, \overrightarrow{A_{m}}\right)$.

Proof. It follows that $\vartheta_{A}\left(M_{\mathcal{R}}\left(\vec{A}_{1}, \ldots, \vec{A}_{m}\right)\right)=$ $\vartheta_{A}\left(m(n+1)-\theta\left(a_{1}, \mathcal{R}\left(\vec{A}_{1}, \ldots, \vec{A}_{m}\right)\right), \ldots, m(n+\right.$ 1) $\left.\left.-\theta\left(a_{n}, \mathcal{R}\left(\overrightarrow{A_{1}}, \ldots, \overrightarrow{A_{m}}\right)\right)\right)\right)$. In addition, we have that $\left.\theta\left(a_{i}, \quad \vartheta_{A}\left(M_{\mathcal{R}}\left(\vec{A}_{1}, \ldots, \vec{A}_{m}\right)\right)\right)\right)<$ $\theta\left(a_{j}, \vartheta_{A}\left(M_{\mathcal{R}}\left(\overrightarrow{A_{1}}, \ldots, \overrightarrow{A_{m}}\right)\right)\right) \Leftrightarrow \pi_{i}\left(M_{\mathcal{R}}\left(\overrightarrow{A_{1}}, \ldots\right.\right.$, $\left.\left.\vec{A}_{m}\right)\right) \quad>\quad \pi_{j}\left(M_{\mathcal{R}}\left(\vec{A}_{1}, \ldots, \vec{A}_{m}\right)\right) \quad \Leftrightarrow$ $m(n+1)-\theta\left(a_{i}, \mathcal{R}\left(\overrightarrow{A_{1}}, \ldots, \vec{A}_{m}\right)\right)$ $m(n+1)-\theta\left(a_{j}, \mathcal{R}\left(\vec{A}_{1}, \ldots, \vec{A}_{m}\right)\right) \quad \Leftrightarrow$ $\theta\left(a_{i}, \mathcal{R}\left(\overrightarrow{A_{1}}, \ldots, \overrightarrow{A_{m}}\right)\right)<\theta\left(a_{j}, \mathcal{R}\left(\vec{A}_{1}, \ldots, \overrightarrow{A_{m}}\right)\right)$. In an analogous way it is possible to prove that $\left.\theta\left(a_{i}, \vartheta_{A}\left(M_{\mathcal{R}}\left(\overrightarrow{A_{1}}, \ldots, \overrightarrow{A_{m}}\right)\right)\right)\right)=$ $\theta\left(a_{j}, \vartheta_{A}\left(M_{\mathcal{R}}\left(\vec{A}_{1}, \ldots, \vec{A}_{m}\right)\right)\right) \quad$ if, and only if, $\quad \theta\left(a_{i}, \mathcal{R}\left(\vec{A}_{1}, \ldots, \vec{A}_{m}\right)\right)=\theta\left(a_{j}, \mathcal{R}\left(\vec{A}_{1}\right.\right.$, $\left.\left.\ldots, \overrightarrow{A_{m}}\right)\right)$. Therefore, $\vartheta_{A}\left(M_{\mathcal{R}}\left(\vec{A}_{1}, \ldots, \overrightarrow{A_{m}}\right)\right)=$ $\mathcal{R}\left(\vec{A}_{1}, \ldots, \vec{A}_{m}\right)$.
Example VI.8. Consider the ranking fusion function $\mathcal{R}_{1}$ and $\overrightarrow{A_{1}}, \overrightarrow{A_{2}}$ and $\overrightarrow{A_{3}}$ of Example VI.5. Then, $\mathcal{R}_{1}\left(\overrightarrow{A_{1}}, \overrightarrow{A_{2}}, \overrightarrow{A_{3}}\right)=\left(\left\{a_{1}, a_{2}\right\},\left\{a_{3}, a_{4}\right\}\right)$. So, $\theta\left(a_{1}, \mathcal{R}_{1}\left(\overrightarrow{A_{1}}, \overrightarrow{A_{2}}, \overrightarrow{A_{3}}\right)\right)=\theta\left(a_{2}, \mathcal{R}_{1}\left(\overrightarrow{A_{1}}, \overrightarrow{A_{2}}, \overrightarrow{A_{3}}\right)\right)=1$ and $\theta\left(a_{3}, \mathcal{R}_{1}\left(\overrightarrow{A_{1}}, \overrightarrow{A_{2}}, \overrightarrow{A_{3}}\right)\right)=\theta\left(a_{4}, \mathcal{R}_{1}\left(\overrightarrow{A_{1}}, \overrightarrow{A_{2}}\right.\right.$, $\left.\left.\overrightarrow{A_{3}}\right)\right)=3$. Therefore, $M_{\mathcal{R}_{1}}\left(\overrightarrow{A_{1}}, \overrightarrow{A_{2}}, \overrightarrow{A_{3}}\right)=$ $(14,14,12,12)$. Hence, $\vartheta_{A}\left(M_{\mathcal{R}_{1}}\left(\overrightarrow{A_{1}}, \overrightarrow{A_{2}}, \overrightarrow{A_{3}}\right)\right)=$ $\vartheta_{A}(14,14,12,12)=\left(\left\{a_{1}, a_{2}\right\},\left\{a_{3}, a_{4}\right\}\right)=$ $\mathcal{R}_{1}\left(\overrightarrow{A_{1}}, \overrightarrow{A_{2}}, \overrightarrow{A_{3}}\right)$.

Finally, we are ready to prove now the result that, jointly with Theorem VI.6 above, establishes the crucial equivalence existing between ranking fusion functions and $A$-score functions.

Theorem VI.9. Let $A=\left\{a_{1}, \ldots, a_{n}\right\}$ be $a$ set of alternatives. Let $\mathcal{R}: \bigcup_{m=1}^{\infty} \wp_{A}^{m} \rightarrow \wp_{A}$ be a ranking fusion function ${ }^{m=1}$ such that if $\theta\left(a_{j}, \mathcal{R}\left(\overrightarrow{A_{1}}, \ldots, \overrightarrow{A_{m}}\right)\right) \leq \theta\left(a_{l}, \mathcal{R}\left(\overrightarrow{A_{1}}, \ldots, \overrightarrow{A_{m}}\right)\right)$ and $\theta\left(a_{j}, \vec{A}_{m+1}\right) \leq \theta\left(a_{l}, \vec{A}_{m+1}\right)$ for some $\vec{A}_{m+1} \in \wp_{A}$ and $j, \bar{l} \in N_{n}$, then it holds true that $\theta\left(a_{j}, \mathcal{R}\left(\vec{A}_{1}, \ldots, \vec{A}_{m+1}\right)\right) \leq \theta\left(a_{l}, \mathcal{R}\left(\vec{A}_{1}, \ldots\right.\right.$, $\left.\left.\vec{A}_{m+1}\right)\right)$. Then, the function $M_{\mathcal{R}}: \bigcup_{m=1}^{\infty} \wp_{A}^{m} \rightarrow N^{n}$ defined by $M_{\mathcal{R}}\left(\vec{A}_{1}, \ldots, \overrightarrow{A_{m}}\right)=(m(n+$ 1) $-\theta\left(a_{1}, \mathcal{R}\left(\vec{A}_{1}, \ldots, \vec{A}_{m}\right)\right), \ldots, m(n+1)-$ $\left.\theta\left(a_{n}, \mathcal{R}\left(\vec{A}_{1}, \ldots, \vec{A}_{m}\right)\right)\right)$ is an A-score function.

Proof. The condition SF1 directly follows from the commutativity of $\mathcal{R}$.

Let us check now the condition SF2: To do so, let $j, l \in N_{n}$. We will prove SF2 by induction in $m$.

When $m=1$, it follows that if $\theta\left(a_{j}, \overrightarrow{A_{1}}\right) \leq$ $\theta\left(a_{l}, \overrightarrow{A_{1}}\right)$ then $\pi_{j}\left(M_{\mathcal{R}}\left(\overrightarrow{A_{1}}\right)\right)=\pi_{j}(m(n+1)-$ $\left.\theta\left(a_{1}, \mathcal{R}\left(\overrightarrow{A_{1}}\right)\right), \ldots, m(n+1)-\theta\left(a_{n}, \mathcal{R}\left(\vec{A}_{1}\right)\right)\right)=$ $\pi_{j}\left(m(n+1)-\theta\left(a_{1}, \overrightarrow{A_{1}}\right), \ldots, m(n+1)-\theta\left(a_{n}, \overrightarrow{A_{1}}\right)\right)=$ $m(n+1)-\theta\left(a_{j}, \overrightarrow{A_{1}}\right) \geq m(n+1)-\theta\left(a_{l}, \overrightarrow{A_{1}}\right)=$ $\pi_{l}\left(M_{\mathcal{R}}\left(\overrightarrow{A_{1}}\right)\right)$.

Now we use the inductive hypothesis, namely if $\theta\left(a_{j}, \vec{A}_{i}\right) \leq \theta\left(a_{l}, \vec{A}_{i}\right)$ for each $i \in N_{m}$ then $\pi_{j}\left(M_{\mathcal{R}}\left(\overrightarrow{A_{1}}, \ldots, \overrightarrow{A_{m}}\right)\right) \geq \pi_{l}\left(M_{\mathcal{R}}\left(\overrightarrow{A_{1}}, \ldots, \overrightarrow{A_{m}}\right)\right)$. The inductive step goes now as follows: If $\theta\left(a_{j}, \overrightarrow{A_{i}}\right) \leq \theta\left(a_{l}, \vec{A}_{i}\right)$ for each $i \in N_{m+1}$ then, by the inductive hypothesis, we have that $\pi_{j}\left(M_{\mathcal{R}}\left(\overrightarrow{A_{1}}, \ldots, \overrightarrow{A_{m}}\right)\right) \geq \pi_{l}\left(M_{\mathcal{R}}\left(\overrightarrow{A_{1}}, \ldots, \overrightarrow{A_{m}}\right)\right)$. So $\pi_{j}\left(m(n+1)-\theta\left(a_{1}, \mathcal{R}\left(\overrightarrow{A_{1}}, \ldots, \overrightarrow{A_{m}}\right)\right), \ldots, m(n+\right.$ 1) $\left.-\theta\left(a_{n}, \mathcal{R}\left(\overrightarrow{A_{1}}, \ldots, \overrightarrow{A_{m}}\right)\right)\right) \geq \pi_{l}(m(n+$ 1) $\quad-\quad \theta\left(a_{1}, \mathcal{R}\left(\vec{A}_{1}, \ldots, \vec{A}_{m}\right)\right), \ldots, m(n \quad+$ 1) $\left.\quad-\quad \theta\left(a_{n}, \mathcal{R}\left(\overrightarrow{A_{1}}, \ldots, \overrightarrow{A_{m}}\right)\right)\right)$ Hence 
$m(n+1)-\theta\left(a_{j}, \mathcal{R}\left(\overrightarrow{A_{1}}, \ldots, \overrightarrow{A_{m}}\right) \geq\right.$ $m(n+1)-\theta\left(a_{l}, \mathcal{R}\left(\overrightarrow{A_{1}}, \ldots, \overrightarrow{A_{m}}\right)\right) . \quad$ Therefore, $\theta\left(a_{j}, \mathcal{R}\left(\overrightarrow{A_{1}}, \ldots, \overrightarrow{A_{m}}\right)\right) \leq \theta\left(a_{l}, \mathcal{R}\left(\overrightarrow{A_{1}}, \ldots, \overrightarrow{A_{m}}\right)\right)$. Since, $\theta\left(a_{j}, \vec{A}_{m+1}\right) \leq \theta\left(a_{l}, \vec{A}_{m+1}\right)$ then by the conditions of the statement imposed to $\mathcal{R}$, we have that $\theta\left(a_{j}, \mathcal{R}\left(\overrightarrow{A_{1}}\right.\right.$, $\left.\left.\ldots, \vec{A}_{m+1}\right)\right) \leq \theta\left(a_{l}, \mathcal{R}\left(\vec{A}_{1}, \ldots, \vec{A}_{m+1}\right)\right)$. Thus $(m+1)(n+1)-\theta\left(a_{j}, \mathcal{R}\left(\vec{A}_{1}, \ldots, \vec{A}_{m+1}\right)\right) \geq(m+$ 1) $(n+1)-\theta\left(a_{l}, \mathcal{R}\left(\overrightarrow{A_{1}}, \ldots, \vec{A}_{m+1}\right)\right)$. Consequently, we conclude that $\pi_{j}\left(M_{\mathcal{R}}\left(\vec{A}_{1}, \ldots, \vec{A}_{m+1}\right)\right) \geq$ $\pi_{l}\left(M_{\mathcal{R}}\left(\vec{A}_{1}, \ldots, \vec{A}_{m+1}\right)\right)$.

Concerning the condition SF3, by Lemma VI.7 and GM3 we have that $\vartheta_{A}\left(M_{\mathcal{R}}\left(\vec{A}_{1}, \ldots, \vec{A}_{m}, \vartheta_{A}\left(M_{\mathcal{R}}\left(\vec{A}_{1}, \ldots, \vec{A}_{m}\right)\right)\right)\right)=$ $\mathcal{R}\left(\vec{A}_{1}, \ldots, \overrightarrow{A_{m}}, \mathcal{R}\left(\vec{A}_{1}, \ldots, \overrightarrow{A_{m}}\right)\right)$ $\mathcal{R}\left(\vec{A}_{1}, \ldots, \overrightarrow{A_{m}}\right)=\vartheta_{A}\left(M_{\mathcal{R}}\left(\overrightarrow{A_{1}}, \ldots, \vec{A}_{m}\right)\right)$.

Finally, the condition SF4 also holds true because, for each $i \in N_{n}$, it follows that $\pi_{i}\left(M_{\mathcal{R}}\left(\vec{A}_{1}, \ldots, \vec{A}_{m}\right)\right) \in\left(N_{m(n+1)-1}-N_{m(n+1)-n}\right)$ and similarly $\quad \pi_{i}\left(M_{\mathcal{R}}\left(\vec{A}_{1}, \ldots, \vec{A}_{m+1}\right)\right) \quad \in$ $\left(N_{(m+1)(n+1)-1} \quad-\quad N_{(m+1)(n+1)-n}\right) . \quad$ Hence it is clear that $\pi_{i}\left(M_{\mathcal{R}}\left(\vec{A}_{1}, \ldots, \overrightarrow{A_{m}}\right)\right)<$ $\pi_{i}\left(M_{\mathcal{R}}\left(\vec{A}_{1}, \ldots, \vec{A}_{m+1}\right)\right)$.

\section{OPTIMALITY ANALYSIS: RANKING FUSION FUNCTIONS INDUCE PARTIAL ORDERS ON THE SET OF ALL THE POSSIBLE RANKINGS}

Suppose that we have at hand different ranking fusion functions, or more generally different methods that, given a finite collection of total preorders defined on a nonempty set, combine them someway, so that the final output is another total order.

Which is the best ranking fusion function, or the optimal method of combination?

Bearing in mind the idea of setting optimality in some theoretical suitable way, a possible formalization that fits well our purposes could be the definition, directly, of some ordering (e.g., a total preorder, a partial order, etc.) on the set of rankings. Once this has been done, a first fusion method could be considered better than a second one when the final rankings got as output of the first method acting on a $k$-tuple of rankings or preferences is better, as regards to the ordering defined on the set of rankings, that the corresponding output ranking furnished by the second method. This fact is a key in the approach we follow in the present manuscript.
In this section we introduce an ordering to compare rankings, or, so-to-say, a "ranking of rankings". As commented in Section I (Objectives), this allows us to find a gateway to the discussion about optimality of aggregation methods.

To do so, first we show how a partial order on the set of all the possible rankings that can be on a finite set $A$ of $n$ alternatives actually gives rise to a ranking fusion function. And, conversely, then we will also show that some suitable kinds of ranking fusion functions induce partial orders on the set of rankings defined on $A$.

To start with, let $A$ be a finite set of $n$ alternatives. It is now possible to define a (partial) order, that we will denote $\preceq$, in order to compare rankings on a finite set $A$ of $n$ alternatives. We proceed as follows: Given two rankings $\overrightarrow{A_{1}}, \overrightarrow{A_{2}}$ on the set $A$, we declare that $\overrightarrow{A_{1}} \preceq \vec{A}_{2}$ if and only if for any alternatives $a_{1}, a_{2} \in \bar{A}$ with $a_{2} \succ_{\vec{A}_{1}} a_{1}$, it holds that $a_{2} \succ_{\vec{A}_{2}} a_{1}$.

We may observe now that the rankings $\vec{A}_{\rho}=$ $(\{\rho(1)\}, \ldots,\{\rho(n)\})$, where $\rho: N_{n} \rightarrow A$ is any bijection, constitute the maximal elements for this order $\preceq$, whereas the ranking $\vec{A}=(A)$ is the only infimum. Indeed, the resulting partially ordered set is an inf-semilattice in the sense of [15, Def. O-1.8].

It is plain that, if $\left(\wp_{A}, \preceq\right)$ is an inf- (sup)semilattice, then the function $\mathcal{R}: \bigcup_{m=1}^{\infty} \wp_{A}^{m} \rightarrow \wp_{A}$ defined by $\mathcal{R}\left(\overrightarrow{A_{1}}, \ldots, \overrightarrow{A_{m}}\right)=\inf \left(\overrightarrow{A_{1}}, \ldots, \overrightarrow{A_{m}}\right)$ is actually a ranking fusion function.

An analogous situation arises if we take $\mathcal{R}\left(\overrightarrow{A_{1}}, \ldots, \overrightarrow{A_{m}}\right)=\sup \left(\overrightarrow{A_{1}}, \ldots, \overrightarrow{A_{m}}\right)$. Nevertheless, these ranking fusion functions are useless when dealing with for some particular inf- (sup-) semilattices. The reason is that in several cases (as it happens with respect to the order $\preceq$ ) they return as output the bottom (top) ranking.

This unpleasant situation can be avoided when the order defined on the collection of rankings is total, as, for example, the following one: $\vec{A} \preceq_{\rho} \vec{B}$ if and only if $\vec{A}=\vec{B}$ or $\theta\left(\rho\left(i_{0}\right), \vec{A}\right)<$ $\theta\left(\rho\left(i_{0}\right), \vec{B}\right)$, where $\rho: N_{n} \rightarrow A$ is a bijection, and $i_{0} \in N_{n}$ is the smallest index such that $\theta\left(\rho\left(i_{0}\right), \vec{A}\right) \neq$ $\theta\left(\rho\left(i_{0}\right), \vec{B}\right)$.

The problem with the ranking fusion function defined above, with respect to the total order $\preceq_{\rho}$, is that it could return as output one of the rankings given as input. And, in addition, this is based on a bijection $\rho$ which can be quite arbitrary. As a matter 
of fact, it is enough that one of the rankings be $\vec{A}_{\rho}=(\rho(1), \ldots, \rho(n))$ for the function $\mathcal{R}$ to lead to as output or final result the ranking $\vec{A}_{\rho}$, even when all the other rankings are equal and coincide with a ranking $\vec{A}$ different from $\vec{A}_{\rho}$. Thus, under these hypotheses $\vec{A}_{\rho}$ should be considered in a sense as the perfect ranking.

At this point we have just seen that partial orders defined on $\wp_{A}$-set of all possible rankings on a finite set $A$ - generate ranking fusion functions. To complete the panorama, we prove now that, conversely, some particular kind of ranking fusion functions can generate a partial order on $\wp_{A}$.

Proposition VII.1. Let $A$ be a finite and nonempty set of $n$ alternatives. Let $\mathcal{R}$ : $\bigcup_{m=1}^{\infty} \wp_{A}^{m} \rightarrow \wp_{A}$ be a ranking fusion function such that the self-distributive law holds, that is: $\mathcal{R}(\vec{A}, \mathcal{R}(\vec{B}, \vec{C}))=\mathcal{R}(\mathcal{R}(\vec{A}, \vec{B}), \mathcal{R}(\vec{A}, \vec{C})) \quad(S D)$. Then the structure $\left(\wp(A), \preceq_{\mathcal{R}}\right)$, where $\vec{A} \preceq_{\mathcal{R}}$ $\vec{B}$ if and only if $\mathcal{R}(\vec{A}, \vec{B})=\vec{A}$ is a partially ordered set (poset). In addition, if $\mathcal{R}$ is also associative when restricted to $m=2$, that is, when $\mathcal{R}(\vec{A}, \mathcal{R}(\vec{B}, \vec{C}))=\mathcal{R}(\mathcal{R}(\vec{A}, \vec{B}), \vec{C})$, then it holds true that $\left(\wp(A), \preceq_{\mathcal{R}}\right)$ is a bounded inf-semillatice.

Proof. First we will prove that $\preceq_{\mathcal{R}}$ is a partial order.

Reflexivity: Since $\mathcal{R}$ is idempotent (i.e., it satisfies the condition GM2), then for each ranking $\vec{A}$ we have that $\mathcal{R}(\vec{A}, \vec{A})=\vec{A}$. Therefore $\vec{A} \preceq_{\mathcal{R}} \vec{A}$ holds true.

Antisymmetry: If $\vec{A} \preceq_{\mathcal{R}} \vec{B}$ and $\vec{B} \preceq_{\mathcal{R}} \vec{A}$ then by the anonymity-neutrality condition GM1, that $\mathcal{R}$ satisfies by definition, we get $\vec{A}=$ $\mathcal{R}(\vec{A}, \vec{B})=\mathcal{R}(\vec{B}, \vec{A})=\vec{B}$.

Transitivity: If $\vec{A} \preceq_{\mathcal{R}} \vec{B}$ and $\vec{B} \preceq_{\mathcal{R}} \vec{C}$ then we have that the following equalities hold true:

(*) $\mathcal{R}(\vec{A}, \vec{B})=\vec{A} ; \quad$ (**) $\mathcal{R}(\vec{B}, \vec{C})=\vec{B}$.

So, by the condition GM1, we have that $\mathcal{R}(\vec{B}, \vec{A})=\vec{A}$ and also $\mathcal{R}(\vec{C}, \vec{B})=\vec{B}$. Therefore, $\mathcal{R}(\vec{A}, \vec{C})=\mathcal{R}(\vec{C}, \mathcal{R}(\vec{A}, \vec{B}))$ (by GM1 and $\left.\left.{ }^{*}\right)\right)=\mathcal{R}(\mathcal{R}(\vec{C}, \vec{A}), \mathcal{R}(\vec{C}, \vec{B}))$ (by $(\mathrm{SD}))=\mathcal{R}(\vec{B}, \mathcal{R}(\vec{A}, \vec{C}))$ (by GM1 and $(* *))=$ $\mathcal{R}(\mathcal{R}(\vec{B}, \vec{A}), \mathcal{R}(\vec{B}, \vec{C}))$ (by $(\mathrm{SD}))=\mathcal{R}(\vec{A}, \vec{B})$ (by GM1, (*) and (**)) $=\vec{A}(b y(*))$. Hence, by definition of $\preceq_{\mathcal{R}}$, we arrive at $\vec{A} \preceq_{\mathcal{R}} \vec{C}$.

Finally we will prove that, for each pair of rankings $(\vec{A}, \vec{B}) \in \wp(A) \times \wp(A)$, their infimum is $\mathcal{R}(\vec{A}, \vec{B})$ : By (SD) and GM2, we see that
$\mathcal{R}(\vec{A}, \vec{B})=\mathcal{R}(\mathcal{R}(\vec{A}, \vec{A}), \vec{B}))=\mathcal{R}(\vec{A}, \mathcal{R}(\vec{A}, \vec{B}))$. Thus, by definition of $\preceq_{\mathcal{R}}$, we get that $\mathcal{R}(\vec{A}, \vec{B}) \preceq_{\mathcal{R}}$ $\vec{A}$. Similarly it is proved that $\mathcal{R}(\vec{A}, \vec{B}) \preceq_{\mathcal{R}} \vec{B}$. Therefore, $\mathcal{R}(\vec{A}, \vec{B})$ is a lower bound of $\vec{A}$ and $\vec{B}$. Now suppose that $\vec{C}$ is another lower bound. Then, once more by definition of $\preceq_{\mathcal{R}}$, we get $\vec{C}=\mathcal{R}(\vec{C}, \vec{A})$ as well as $\vec{C}=\mathcal{R}(\vec{C}, \vec{B})$. So, by SD jointly with the condition GM2 of idempotence, we obtain $\vec{C}=\mathcal{R}(\vec{C}, \vec{C})=\mathcal{R}(\mathcal{R}(\vec{C}, \vec{A}), \mathcal{R}(\vec{C}, \vec{B}))=$ $\mathcal{R}(\vec{C}, \mathcal{R}(\vec{A}, \vec{B}))$. Therefore, again by definition of $\preceq_{\mathcal{R}}, \vec{C} \preceq_{\mathcal{R}} \mathcal{R}(\vec{A}, \vec{B})$. Hence, $\mathcal{R}(\vec{A}, \vec{B})$ is the infimum of $\vec{A}$ and $\vec{B}$.

\section{Illustrative examples}

Our aim when choosing the examples introduced at the end of this Section VI is just to show how to use ranking fusion functions and $A$-score functions to find maximal elements on rankings defined on a finite set $A$. Above all, at this stage we want to be didactic and explanatory. Bearing this in mind, we have decided not to search for sophisticated situations, nor even algorithms. Thus, the examples given here may perhaps look as naive, but they are easy-to-understand, and the intention behind these examples is to illustrate step-by- step how the concepts previously defined work in practice. We want to explain in a clear and understandable way -even if the examples look simple- how ranking fusion functions give rise to partial orders on the set of all possible rankings on a finite set $A$, and how to use this fact to search for optimality among rankings.

Indeed, some of the examples furnished here had already appeared in this literature: the first one may be seen in [11] and [17], whereas the second one appears in [21]. Incidentally, we point out that the aggregating procedures used by the experts involved in both examples are quite diverse, as mentioned in Table VI and Table XI below. In fact, the preference relations used may even be Atanassov's intuitionistic multiplicative ones, as in the second example. However, we insist on this, our aim here is to show the scope of the processes described before.

First example (See [11], [17]).

Suppose that an investor intends to invest part of her/his capital in a company. By means of a suitable market analysis the investor reduces the spectrum of possible companies into six, namely: 
TABLE III

ASSESSMENT OF EXPERT $e_{1}$.

\begin{tabular}{|l|llllll|}
\hline$e_{1}$ & $a_{1}$ & $a_{2}$ & $a_{3}$ & $a_{4}$ & $a_{5}$ & $a_{6}$ \\
\hline$x_{1}$ & 0.7 & 0.8 & 0.6 & 0.7 & 0.5 & 0.9 \\
$x_{2}$ & 0.8 & 0.6 & 0.9 & 0.7 & 0.6 & 0.7 \\
$x_{3}$ & 0.5 & 0.4 & 0.8 & 0.3 & 0.8 & 0.8 \\
$x_{4}$ & 0.6 & 0.7 & 0.6 & 0.7 & 0.8 & 0.6 \\
$x_{5}$ & 0.9 & 0.8 & 0.4 & 0.7 & 0.7 & 0.8 \\
$x_{6}$ & 0.8 & 0.3 & 0.7 & 0.7 & 0.6 & 0.7 \\
\hline
\end{tabular}

TABLE IV

ASSESSMENT OF EXPERT $e_{2}$.

\begin{tabular}{|l|llllll|}
\hline$e_{2}$ & $a_{1}$ & $a_{2}$ & $a_{3}$ & $a_{4}$ & $a_{5}$ & $a_{6}$ \\
\hline$x_{1}$ & 0.6 & 0.8 & 0.5 & 0.6 & 0.4 & 0.8 \\
$x_{2}$ & 0.7 & 0.6 & 0.8 & 0.6 & 0.7 & 0.7 \\
$x_{3}$ & 0.7 & 0.6 & 0.8 & 0.7 & 0.8 & 0.8 \\
$x_{4}$ & 0.6 & 0.7 & 0.5 & 0.6 & 0.8 & 0.7 \\
$x_{5}$ & 0.7 & 0.8 & 0.7 & 0.7 & 0.6 & 0.8 \\
$x_{6}$ & 0.6 & 0.4 & 0.8 & 0.7 & 0.6 & 0.7 \\
\hline
\end{tabular}

- A chemical company, denoted by $x_{1}$.

- A food company denoted by $x_{2}$.

- A computer company denoted by $x_{3}$.

- A car company denoted by $x_{4}$.

- A furniture company denoted by $x_{5}$.

- A pharmaceutical company denoted by $x_{6}$.

The investor is helped by a group of three experts or decision makers (namely $e_{1}, e_{2}$ and $e_{3}$ ) with the following weights $\omega=(0.3,0.3,0.4)$. This group of experts establish that six attributes will be used to evaluate the investments.

The benefit attributes are: $a_{1}$ ) benefits in the short term; $a_{2}$ ) benefits in the middle term; $a_{3}$ ) benefits in the long term. And the cost attributes are: $a_{4}$ ) risk of the investment; $a_{5}$ ) difficulty of the investment, and $a_{6}$ ) other unfavorable factors on the investment.

Tables III, IV and V describe the assesses of the experts about how much the investment has to do with each attribute. In other words, each of them constitutes the decision matrix of the corresponding expert.

In addition, Table VI, shows the ranking obtained as output after using different fusion methods, for this particular decision making example. Notice here that all those rankings finally got are pairwise different.

Applying now to these rankings the $A$-score

TABLE V

ASSESSMENT OF EXPERT $e_{3}$.

\begin{tabular}{|l|llllll|}
\hline$e_{3}$ & $a_{1}$ & $a_{2}$ & $a_{3}$ & $a_{4}$ & $a_{5}$ & $a_{6}$ \\
\hline$x_{1}$ & 0.7 & 0.6 & 0.6 & 0.6 & 0.4 & 0.7 \\
$x_{2}$ & 0.7 & 0.6 & 0.7 & 0.6 & 0.6 & 0.7 \\
$x_{3}$ & 0.6 & 0.5 & 0.8 & 0.5 & 0.8 & 0.8 \\
$x_{4}$ & 0.6 & 0.7 & 0.7 & 0.5 & 0.8 & 0.6 \\
$x_{5}$ & 0.7 & 0.8 & 0.6 & 0.7 & 0.6 & 0.8 \\
$x_{6}$ & 0.4 & 0.5 & 0.9 & 0.7 & 0.6 & 0.6 \\
\hline
\end{tabular}

TABLE VI

SUMMARY OF THE RANKINGS OBTAINED IN [10], [11], [17].

\begin{tabular}{|l|l|c|l|}
\hline & Methods & source & \multicolumn{1}{|c|}{ Ranking } \\
\hline$\vec{A}_{1}$ & Maximum & {$[17]$} & $x_{2}>x_{4}>x_{5}>x_{1}>x_{3}>x_{6}$ \\
$\vec{A}_{2}$ & Minimum & {$[17]$} & $x_{3} \sim x_{5}>x_{1}>x_{4}>x_{2}>x_{6}$ \\
$\vec{A}_{3}$ & NHD & {$[17]$} & $x_{5}>x_{2}>x_{3}>x_{4}>x_{1}>x_{6}$ \\
$\vec{A}_{4}$ & WHD & {$[17]$} & $x_{5}>x_{3}>x_{2}>x_{4}>x_{6}>x_{1}$ \\
$\vec{A}_{5}$ & Step-IOWAD & {$[17]$} & $x_{5}>x_{4}>x_{6}>x_{2}>x_{3}>x_{1}$ \\
$\vec{A}_{6}$ & Hurwicz & {$[17]$} & $x_{3}>x_{2}>x_{6}>x_{4}>x_{1}>x_{5}$ \\
$\vec{A}_{7}$ & OWAD & {$[17]$} & $x_{5}>x_{3}>x_{2}>x_{4}>x_{1}>x_{6}$ \\
$\vec{A}_{8}$ & AOWAD & {$[17]$} & $x_{5}>x_{2}>x_{4}>x_{3}>x_{6}>x_{1}$ \\
$\vec{A}_{9}$ & IOWAD & {$[17]$} & $x_{5}>x_{3}>x_{2}>x_{1}>x_{4}>x_{6}$ \\
$\vec{A}_{10}$ & AIOWAD & {$[17]$} & $x_{5}>x_{2}>x_{6}>x_{4}>x_{3}>x_{1}$ \\
$\vec{A}_{11}$ & Median-IOWAD & {$[17]$} & $x_{5}>x_{1}>x_{6}>x_{2}>x_{4}>x_{3}$ \\
$\vec{A}_{12}$ & Olympic-IOWAD & {$[17]$} & $x_{5}>x_{4}>x_{1}>x_{2}>x_{3}>x_{6}$ \\
$\vec{A}_{13}$ & $W A_{\omega}^{O} O_{0.5 M}^{3}$ & {$[10]$} & $x_{5}>x_{2}>x_{1}>x_{4}>x_{3}>x_{6}$ \\
$\vec{A}_{14}$ & $W A_{\omega}^{O_{2}^{3}}$ & {$[10]$} & $x_{2}>x_{1}>x_{5}>x_{4}>x_{3}>x_{6}$ \\
$\vec{A}_{15}$ & $W A_{w}^{P}$ & {$[11]$} & $x_{2} \sim x_{3}>x_{6}>x_{4}>x_{1}>x_{5}$ \\
\hline
\end{tabular}

function $M_{1}$ introduced in the Example VI.5, we obtain:

- $\pi_{1}\left(M_{1}\left(\overrightarrow{A_{1}}, \ldots, \overrightarrow{A_{15}}\right)\right)=90-(4+3+5+6+$ $6+5+5+6+4+6+2+3+3+2+5)=25$

- $\pi_{2}\left(M_{1}\left(\overrightarrow{A_{1}}, \ldots, \overrightarrow{A_{15}}\right)\right)=90-(1+5+2+3+$ $4+2+3+2+3+2+4+4+2+1+1)=56$

- $\pi_{3}\left(M_{1}\left(\overrightarrow{A_{1}}, \ldots, \overrightarrow{A_{15}}\right)\right)=90-(5+1+3+2+$ $5+1+2+4+2+5+6+5+5+5+1)=38$ - $\pi_{4}\left(M_{1}\left(\overrightarrow{A_{1}}, \ldots, \overrightarrow{A_{15}}\right)\right)=90-(2+4+4+4+$ $2+4+4+3+5+4+5+2+4+4+4)=35$

- $\pi_{5}\left(M_{1}\left(\overrightarrow{A_{1}}, \ldots, \overrightarrow{A_{15}}\right)\right)=90-(3+1+1+1+$ $1+6+1+1+1+1+1+1+1+3+6)=61$

- $\pi_{6}\left(M_{1}\left(\overrightarrow{A_{1}}, \ldots, \overrightarrow{A_{15}}\right)\right)=90-(6+6+6+5+$ $3+3+6+5+6+3+3+6+6+6+3)=17$.

That is, $\quad M_{1}\left(\overrightarrow{A_{1}}, \ldots, \overrightarrow{A_{15}}\right) \quad=$ $(25,56,38,35,61,17)$. Hence $\mathcal{R}_{1}\left(\overrightarrow{A_{1}}, \ldots, \overrightarrow{A_{15}}\right)=$ $\left(\left\{x_{5}\right\},\left\{x_{2}\right\},\left\{x_{3}\right\},\left\{x_{4}\right\},\left\{x_{1}\right\},\left\{x_{6}\right\}\right)$. Thus, the fusion of the rankings that appear in Table VI, with respect to the the first method considered in Example VI.5, is the following output ranking: $x_{5}>x_{2}>x_{3}>x_{4}>x_{1}>x_{6}$. Instead, if we use now the $A$-score function $M_{2}$ introduced through the Example VI.5 to those rankings in Table VI, we obtain:

- $\pi_{1}\left(M_{2}\left(\overrightarrow{A_{1}}, \ldots, \overrightarrow{A_{15}}\right)\right)=0+3+5+5+3+9=25$

- $\pi_{2}\left(M_{2}\left(\overrightarrow{A_{1}}, \ldots, \overrightarrow{A_{15}}\right)\right)=12+0+9+12+4+13=50$

- $\pi_{3}\left(M_{2}\left(\overrightarrow{A_{1}}, \ldots, \overrightarrow{A_{15}}\right)\right)=10+5+0+7+2+12=36$

- $\pi_{4}\left(M_{2}\left(\overrightarrow{A_{1}}, \ldots, \overrightarrow{A_{15}}\right)\right)=10+3+8+0+3+11=35$

- $\pi_{5}\left(M_{2}\left(\overrightarrow{A_{1}}, \ldots, \overrightarrow{A_{15}}\right)\right)=12+11+12+12+0+13=$ 60

- $\pi_{6}\left(M_{2}\left(\overrightarrow{A_{1}}, \ldots, \overrightarrow{A_{15}}\right)\right)=6+2+3+4+2+0=17$.

That is, $M_{2}\left(\overrightarrow{A_{1}}, \ldots, \overrightarrow{A_{15}}\right)=(25,50$, $36,35,60,17)$ and consequently we arrive at $\mathcal{R}_{2}\left(\overrightarrow{A_{1}}, \ldots, \overrightarrow{A_{15}}\right)=$ $\left(\left\{x_{5}\right\},\left\{x_{2}\right\},\left\{x_{3}\right\},\left\{x_{4}\right\},\left\{x_{1}\right\},\left\{x_{6}\right\}\right)$. Thus, the 
TABLE VII

ASSESSMENT OF THE STUDENT $e_{1}$.

\begin{tabular}{|c|cccc|}
\hline$e_{1}$ & $x_{1}$ & $x_{2}$ & $x_{3}$ & $x_{4}$ \\
\hline$x_{1}$ & - & {$[0.01,0.675]$} & {$[0.025,0.9]$} & {$[0.04375,0.9]$} \\
$x_{2}$ & {$[0.325,0.99]$} & - & {$[0.015625,0.7875]$} & {$[0.025,0.7875]$} \\
$x_{3}$ & {$[0.1,0.975]$} & {$[0.2125,0.984375]$} & - & {$[0.025,0.9]$} \\
$x_{4}$ & {$[0.1,0.95625]$} & {$[0.2125,0.975]$} & {$[0.1,0.975]$} & - \\
\hline
\end{tabular}

TABLE VIII

ASSESSMENT OF THE STUDENT $e_{2}$.

\begin{tabular}{|c|cccc|}
\hline$e_{2}$ & $x_{1}$ & $x_{2}$ & $x_{3}$ & $x_{4}$ \\
\hline$x_{1}$ & - & {$[0.025,0.7875]$} & {$[0.015625,0.7875]$} & {$[0.025,0.9]$} \\
$x_{2}$ & {$[0.2125,0.975]$} & - & {$[0.01,0.675]$} & {$[0.015625,0.7875]$} \\
$x_{3}$ & {$[0.2125,0.984375]$} & {$[0.325,0.99]$} & - & {$[0.04375,0.9]$} \\
$x_{4}$ & {$[0.1,0.975]$} & {$[0.2125,0.84375]$} & {$[0.1,0.95625]$} & - \\
\hline
\end{tabular}

fusion of the rankings that appear in Table VI, as regards the second method in Example VI.5, is again the same ranking that the one obtained above by means of the first fusion function, namely $x_{5}>x_{2}>x_{3}>x_{4}>x_{1}>x_{6}$.

\section{Second example (See [21]).}

Four university students share a house, where they intend to have broadband internet connection installed. There are four available options to choose from, which are furnished by four internet service providers: $x_{1}: 1 \mathrm{Mb} / \mathrm{s}$ broadband; $x_{2}: 2 \mathrm{Mb} / \mathrm{s}$ broadband; $x_{3}: 3 \mathrm{Mb} / \mathrm{s}$ broadband; $x_{4}: 4 \mathrm{Mb} / \mathrm{s}$ broadband.

The internet service and its corresponding monthly bill will be shared by the four students $\left\{e_{1}, e_{2}, e_{3}, e_{4}\right\}$. They decide to perform a multiexpert decision making problem, considering the weight vector $w=(0.3,0.3,0.2,0.2)$. The students determine their preference relations in an independent and anonymous way. In [21] it was considered the use of Atanassov's intuitionistic multiplicative preference relations in the range $\left[\frac{1}{9}, 9\right]$ and in [6] those preference relations were transformed, via the linear transformation $f(x)=\frac{80 x+1}{9}$, in the interval-valued fuzzy preference relations shown in the Tables VII, VIII, IX and X.

The Table XI, shows the rankings obtained from different methods for this decision making example.

Applying the $A$-score function $M_{1}$ introduced by means of the Example VI.5 to these rankings, we

TABLE IX

ASSESSMENT OF THE STUDENT $e_{3}$.

\begin{tabular}{|c|cccc|}
\hline$e_{3}$ & $x_{1}$ & $x_{2}$ & $x_{3}$ & $x_{4}$ \\
\hline$x_{1}$ & - & {$[0.2125,0.975]$} & {$[0.025,0.7875]$} & {$[0.1,0.975]$} \\
$x_{2}$ & {$[0.025,0.7875]$} & - & {$[0.015625,0.675]$} & {$[0.01,0.675]$} \\
$x_{3}$ & {$[0.2125,0.975]$} & {$[0.325,0.984375]$} & - & {$[0.1,0.95625]$} \\
$x_{4}$ & {$[0.025,0.9]$} & {$[0.325,0.99]$} & {$[0.04375,0.9]$} & - \\
\hline
\end{tabular}

TABLE X

ASSESSMENT OF THE STUDENT $e_{4}$.

\begin{tabular}{|c|cccc|}
\hline$e_{4}$ & $x_{1}$ & $x_{2}$ & $x_{3}$ & $x_{4}$ \\
\hline$x_{1}$ & - & {$[0.025,0.9]$} & {$[0.04375,0.9]$} & {$[0.04375,0.95625]$} \\
$x_{2}$ & {$[0.1,0.975]$} & - & {$[0.01,0.5625]$} & {$[0.015625,0.675]$} \\
$x_{3}$ & {$[0.1,0.95625]$} & {$[0.4375,0.99]$} & - & {$[0.04375,0.9]$} \\
$x_{4}$ & {$[0.04375,0.95625]$} & {$[0.325,0.984375]$} & {$[0.1,0.95625]$} & - \\
\hline
\end{tabular}

TABLE XI

SUMMARY OF THE RANKINGS OBTAINED IN [6], [21].

\begin{tabular}{|c|l|c|c|}
\hline & Methods & source & Ranking \\
\hline$\vec{A}_{1}$ & $\preceq X Y$ & {$[6]$} & $x_{4}>x_{3}>x_{1}>x_{2}$ \\
$\vec{A}_{2}$ & $\preceq_{\text {Lex } 1}$ & {$[6]$} & $x_{3}>x_{4}>x_{1}>x_{2}$ \\
$\vec{A}_{3}$ & $\varliminf_{\text {Lex } 2}$ & {$[6]$} & $x_{4}>x_{3}>x_{1}>x_{2}$ \\
$\vec{A}_{4}$ & $\preceq_{1}, \frac{2}{3}$ & {$[6]$} & $x_{3}>x_{4}>x_{1}>x_{2}$ \\
$\vec{A}_{5}$ & Approach I & {$[21]$} & $x_{3}>x_{4}>x_{1}>x_{2}$ \\
$\vec{A}_{6}$ & Approach IIa & {$[21]$} & $x_{3}>x_{4}>x_{1}>x_{2}$ \\
$\vec{A}_{7}$ & Approach IIb & {$[21]$} & $x_{4}>x_{3}>x_{1}>x_{2}$ \\
\hline
\end{tabular}

obtain:

- $\pi_{1}\left(M_{1}\left(\overrightarrow{A_{1}}, \ldots, \overrightarrow{A_{7}}\right)\right)=28-(3+3+3+3+$ $3+3+3)=7$

- $\pi_{2}\left(M_{1}\left(\overrightarrow{A_{1}}, \ldots, \overrightarrow{A_{7}}\right)\right)=28-(4+4+4+4+$ $4+4+4)=0$

- $\pi_{3}\left(M_{1}\left(\overrightarrow{A_{1}}, \ldots, \overrightarrow{A_{7}}\right)\right)=28-(2+1+2+1+$ $1+1+2)=18$

- $\pi_{4}\left(M_{1}\left(\overrightarrow{A_{1}}, \ldots, \overrightarrow{A_{7}}\right)\right)=28-(1+2+1+2+$ $2+2+1)=17$.

That is, $M_{1}\left(\overrightarrow{A_{1}}, \ldots, \overrightarrow{A_{7}}\right)=(7,0,18,17)$. Hence $\mathcal{R}_{1}\left(\overrightarrow{A_{1}}, \ldots, \overrightarrow{A_{7}}\right)=\left(\left\{x_{3}\right\},\left\{x_{4}\right\},\left\{x_{1}\right\},\left\{x_{2}\right\}\right)$. Thus, the application of the ranking fusion function $\mathcal{R}_{1}$, i.e. the first method in Example VI.5, to the rankings in the Table VI is the following: $x_{3}>x_{4}>x_{1}>x_{2}$.

Now, applying the $A$-score function $M_{2}$ of the Example VI.5 to this ranking, we get:

- $\pi_{1}\left(M_{2}\left(\overrightarrow{A_{1}}, \ldots, \overrightarrow{A_{7}}\right)\right)=0+7+0+0=7$
- $\pi_{2}\left(M_{2}\left(\overrightarrow{A_{1}}, \ldots, \overrightarrow{A_{7}}\right)\right)=0+0+0+0=0$
- $\pi_{3}\left(M_{2}\left(\overrightarrow{A_{1}}, \ldots, \overrightarrow{A_{7}}\right)\right)=7+7+0+4=18$
- $\pi_{4}\left(M_{2}\left(\overrightarrow{A_{1}}, \ldots, \overrightarrow{A_{7}}\right)\right)=7+7+3+0=17$.

That is, $\quad M_{2}\left(\vec{A}_{1}, \ldots, \vec{A}_{7}\right)$ = $\left(\left\{x_{3}\right\},\left\{x_{4}\right\},\left\{x_{1}\right\},\left\{x_{2}\right\}\right)$. Thus, the fusion of the rankings in the Table XI, with respect to the the second method that arises in Example VI.5, namely the ranking fusion function $\mathcal{R}_{2}$, is exactly the same as the one got with the previous one, namely $x_{3}>x_{4}>x_{1}>x_{2}$.

Observe, that only two different rankings arise as obtained by using the methods in the Table XI: $x_{4}>x_{3}>x_{1}>x_{2}$ (thrice) and $x_{3}>x_{4}>x_{1}>$ $x_{2}$ (four times). Thus both ranking fusion functions finally lead to the ranking which was the mostly determined by the methods in Table XI. 


\section{FINAL REMARKS}

The definition of suitable rankings on a set of alternatives is crucial in many real-world decision making problems. There is a very large number of methods proposed in the literature, considering several aspects of the decision problems: multiple attributes or criteria (with weights or without weights), preference relations and decision matrices with several scales of evaluation $([0,1]$, interval numbers, hesitant numbers, fuzzy numbers, etc.), with incomplete information, etc. (see [19], [22], [23]).

From the point of view of Social Choice, no method could be considered optimal, due to the famous Arrow's impossibility theorem (see [16]). But in a real-life situation of Decision-Making, the aggregations of preferences, opinions or assessments should compulsorily be done. This leads us to search for a good definition of ranking fusion function, that could be acceptable in a broad set of approaches, not only in Decision-Making.

Thus, we have taken into account a recent normative research made in [7] to get an axiomatic new setting that could be understood as a new framework to understand what an abstract media is, valid for a wide and multidisciplinary set of disciplines that includes Information Sciences and, in particular., aggregation of rankings. In the present paper we have adopted (see Definition IV.1) a less restrictive variation of the concept of a mean launched in [7].

Unfortunately, there is no general way to evaluate the quality of the rankings provided by the different methods used. That is, when different methods of aggregation are implemented, we should also compare them, looking for a method that could be considered optimal in a sense. Nevertheless, the idea of optimality can be quite subjective. For instance, a fusion method that is computationally faster than many others could actually be bad or unacceptable in real-life situations. Since the decision maker usually needs to arrive at just one final (output) ranking, the following natural question arises: which one of the different rankings obtained by the application of different methods must she/he use?

This paper proposes some general criteria. On the one hand, our proposal is to consider ranking aggregation methods which fit well to the new abstract definition of a general mean. These are called ranking fusion functions. Also, we introduce here, axiomatically defined, the abstract notion of an $A$-score function and establish its equivalence with the previous concept of a ranking fusion function.

Fortunately, in this new setting different aggregation methods can actually be compared someway. A formal setting to cope with these comparisons is to define a suitable ordering on the rankings, and in this line we prove that suitable ranking fusion functions induce a partial order on the set of all possible rankings that may be defined on a finite set $A$.

Therefore, an aggregation method could be declared to be better than another one, as regards the ordering implemented on the set of all rankings, if the final (output) rankings to which the aggregation method leads to is maximal with respect to the partial order induced by the ranking fusion function considered a priori.

In future works we would like to study which ones, from those classical ranking aggregation methods already introduced in the literature, are actually a ranking fusion function in the sense of Definition IV.1. Indeed, several of the aggregation methods proposed in the literature are based on distances functions defined on sets of rankings (e.g. [1], [13], [14]). So we may study the obtention of suitable ranking fusion functions based on distances. Finally, since the present paper is mainly theoretical (in spite of establishing a new basis to deal with rankings), another task for next future is to use these methods in more practical situations, so writing new pieces of research.

\section{CONCLUSION}

In order to implement suitable aggregation methods for rankings, preferences or opinions, it is crucial to look at different approaches, trying to find a suitable definition. In this paper we have adopted a weakening of the concept of a mean introduced in [7]. It suitably links Social Choice and DecisionMaking approaches. Thus we have introduced the concepts of a ranking fusion function and a score function in abstract, as general means. Finally, the decision maker should also dispose at hand of some idea of optimality, in order to chose some particular method of fusion of rankings from a wide set of aggregation methods. With this aim we have shown that ranking fusion functions give rise to a partial order on the set of all rankings defined on a finite 
set $A$. This constitutes a new device that can be used in order to compare different fusion methods.

The contents of the present paper may now be summarized as follows, pointing out the main takehome messages:

1.- This is a theoretical paper. Because of the impossibility theorems arising in Social Choice, as well as the necessity of aggregating rankings encountered in Decision-Making, a new axiomatic setting is introduced, based on the abstract concept of a general mean.

2.- Ranking fusion functions are defined under that setting of general means. Some examples of ranking fusion functions are described. Then, the concept of a score functions is defined axiomatically and a key equivalence between ranking fusion functions and score functions is shown.

3.- It is shown a close relationship between partial orders on the set of all rankings defined on a finite set $A$, and the family of the ranking fusion functions on $A$. By means of those partial orders we may compare aggregation methods, in the search for optimality.

\section{ACKNOWLEDGMENTS}

This work is partially supported by the research projects ECO2015-65031-R, MTM2015-63608-P (MINECO/ AEI-FEDER, UE), TIN2016-77356$\mathrm{P}$ (MINECO/ AEI-FEDER, UE), PID2019108392GB-I00 (AEI/10.13039/501100011033),

and Brazilian National Council for Scientific and Technological Development CNPq (Proc. 307781/2016-0). Thanks are given to an editor and three anonymous referees for their valuable suggestions.

\section{REFERENCES}

[1] J.A. Aledo, J.A. Gámez, D. Molina: Using extension sets to aggregate partial rankings in a flexible setting. Applied Mathematics and Computation, 290: 208-223, 2016.

[2] K.J. Arrow: Social Coice and Individual Values. Wiley, New York, 1951.

[3] E. Barrenechea, J. Fernandez, M. Pagola, F. Chiclana, H. Bustince: Construction of interval-valued fuzzy preference relations from ignorance functions and fuzzy preference relations. Application to decision making. Knowledge-Based Systems, 58: 33-44, 2014.

[4] D. Ben-Arieh, Z. Chen: Linguistic-labels aggregation and consensus measure for autocratic decision making using group recommendations. IEEE Trans. on Systems, Man, and Cybernetics - Part A: Systems and Humans, 36(3): 558-568, 2006.
[5] H. Bustince, E. Barrenechea, T. Calvo, S. James, G. Beliakov: Consensus in multi-expert decision making problems using penalty functions defined over a Cartesian product of lattices. Information Fusion, 17: 56-64,2014.

[6] H. Bustince, M. Galar, B. Bedregal, A. Kolesárová, R. Mesiar: A new approach to interval-valued Choquet integrals and the problem of ordering in interval-valued fuzzy set applications. IEEE Transactions on Fuzzy Systems, 21(6): 1150-1162, 2013.

[7] M.J. Campión, J.C. Candeal, R.G. Catalán, A. Giarlotta, S. Greco, E. Induráin, J. Montero: An axiomatic approach to finite means. Information Sciences 457-458: 12-28, 2018.

[8] G. Chichilnisky, G.M. Heal: Necessary and sufficient conditions for a resolution of the social choice paradox. Journal of Economic Theory 31(1): 68-87, 1983.

[9] F.Y.L. Chin, X. Deng, Q. Fang, S. Zhu: Approximate and dynamic rank aggregation. Theoretical Computer Science, 325: 409-424, 2004

[10] I.A. da Silva, B.C. Bedregal, H. Bustince: Weighted average operators generated by n-dimensional overlaps and an application in decision making. In Proceeding of joint conference 16th World Congress of the International Fuzzy Systems Association (IFSA) and 9th Conference of the European Society for Fuzzy Logic and Technology (EUSFLAT), Gijón 2015, 1473-1478.

[11] I.A. da Silva, B.C. Bedregal, C.G. da Costa, E. Palmeira, M.P. da Rocha: Pseudo-uninorms and Atanassov's intuitionistic pseudo-uninorms. Journal of Intelligent and Fuzzy Systems, 29(1): 267-281, 2015.

[12] I.A. da Silva, B.C. Bedregal, R.H.N. Santiago: On admissible total orders for interval-valued Atanassov's intuitionistic fuzzy membership degrees. Fuzzy Information and Engineering, 8(2): 169-182, 2016.

[13] G. Dahl, H. Minken: A note on permutations and rank aggregation. Mathematical and Computer Modelling 52: 380-385, 2010.

[14] R. Fagin, R. Kumar, D. Sivakumar, M. Mahdian, E. Vee: Comparing and Aggregating Rankings with Ties. In: Proc. 2004 ACM Symposium on Principles of Database Systems (PODS'04), 47-58.

[15] G. Gierz, K.H. Hofmann, K. Keimel, J.D. Lawson, M. Mislove, D.S. Scott: Continuous Lattices and Domains. Cambridge University Press, Cambridge, 2003.

[16] J.S. Kelly: Social Choice Theory. An Introduction. SpringerVerlag, Berlin, 1988.

[17] J.M. Merigó, M. Casanova: Decision-making with distance measures and induced aggregation operators. Computer \& Industrial Engineering, 60: 66-76, 2011.

[18] D. Paternain, A. Jurío, E. Barrenechea, H. Bustince, B.R.C. Bedregal, E. Szmidt: An alternative to fuzzy methods in decision-making problems. Expert Systems with Applications, 39(9): 7729-7735, 2012.

[19] W. Pedrycz, P. Ekel, R. Parreiras: Fuzzy Multicriteria DecisionMaking: Models, Methods and Applications. John Wiley \& Sons, Ltd., 2011.

[20] H. Shachnai, L. Zhang, T. Matsui: A note on generalized rank aggregation. Information Processing Letters, 109: 647651, 2009.

[21] Z.S. Xu: Priority weight intervals derived from intuitionistic multiplicative preference relations. IEEE Trans. on Fuzzy Systems, 21(4): 642-654, 2013.

[22] H. Yano: Interactive Multiobjective Decision Making Under Uncertainty. CRC Press, 2017.

[23] B. I. Yatsalo, L. Martínez: Fuzzy rank acceptability analysis: a confidence measure of ranking fuzzy numbers. IEEE Transactions on Fuzzy Systems 26 (6), 3579-3593, 2018. 